\title{
新型异甜菊醇衍生物的合成、表征及生物活性研究
}

\author{
郭永泰 ${ }^{a}$ \\ 侯熙彦 $b$ \\ 郑昌吉 $c$ \\ 赵春晖 $d$ \\ 金宇婷 $a$ \\ 高雪琴 $a$ \\ 王 倩 ${ }^{a}$ \\ 孙蕾 ${ }^{a}$ \\ 赵龙铉*, $a, d$ \\ 朴 花*,e \\ ( ${ }^{a}$ 辽宁师范大学化学化工学院 辽宁大连 116029) \\ $\left({ }^{b}\right.$ 大连民族大学生命科学学院 辽宁大连 116600) \\ ( ${ }^{c}$ 延边大学药学院 吉林延吉 133002) \\ ( ${ }^{d}$ 辽宁师范大学 生物技术与分子药物研发辽宁省重点实验室 辽宁大连 116029) \\ ( ${ }^{e}$ 大连医科大学基础医学院 辽宁大连 116044)
}

\begin{abstract}
摘要 以异甜菊醇为先导化合物, 通过连接片段分别在 C-16 和 C-19 位引入吗啉和哌嗪结构, 并对 D 环进行结构改造, 设计合成了 15 种未见文献报道的异甜菊醇衍生物, 并观察其对人皮肤鳞癌细胞(Colo-16)和人肺腺癌细胞(A549)抑制作 用. 生物活性结果显示, 有 4 种化合物对人皮肤鳞状癌细胞(Colo-16)的抑制活性明显优于阳性对照物 5-氟尿嘧啶. 同时 观察其对大肠杆菌 (E. coli 1924)、金黄色葡萄球菌(S. aureus 4220)、变形链球菌(S. mutans 3289)、而甲氧西林金黄色葡 萄球菌(MRSA 3167)和耐喹诺酮金黄色葡萄球菌( $Q R S A 3505)$ 的抑制作用，结果显示，有 6 种化合物对 S. mutans 3289 的 最小抑菌浓度(MIC)为 $8 \mu \mathrm{g} / \mathrm{mL}$ ，与阳性对照物氯霉素相当; $16 \beta$ - $O$-[5-氧亚基-5-(4-甲基-1-哌嗪基)-戊酰基]-19-贝叶酸乙 酯(IS-12b)对 MRSA 3167 的最小抑菌浓度(MIC)为 $8 \mu \mathrm{g} / \mathrm{mL}$ ，与阳性对照物氯霉素相当.
\end{abstract}

关键词 异甜菊醇; 细胞毒活性; 抑菌活性

\section{Synthesis, Characteristics and Bioactivities of Novel Isosteviol Derivatives}

\author{
Guo, Yongtai $^{a} \quad$ Hou, Xiyan $^{b} \quad$ Zheng, Changji $^{c} \quad$ Zhao, Chunhui $^{d} \quad$ Jin, Yuting $^{a}$ \\ Gao, Xueqin ${ }^{a} \quad$ Wang, Qian $^{a} \quad$ Sun, Lei ${ }^{a} \quad$ Zhao, Longxuan*,a,b Piao, Hua ${ }^{*, e}$ \\ ( ${ }^{a}$ School of Chemistry and Chemical Engineering, Liaoning Normal University, Dalian, Liaoning 116029) \\ ( ${ }^{b}$ College of Life Science, Dalian Nationalities University, Dalian, Liaoning 116600) \\ ( ${ }^{c}$ College of Pharmacy, Yanbian University, Yanji, Jilin 133002) \\ ( ${ }^{d}$ Liaoning Provincial Key Laboratory of Biotechnology and Drug Discovery, Liaoning Normal University, \\ Dalian, Liaoning 116029) \\ ( ${ }^{e}$ Department of Physiology, College of Basic Medical Sciences, Dalian Medical University, Dalian, Liaoning 116044)
}

\begin{abstract}
Fifteen novel isosteviol derivatives were designed and synthesized by introducing morpholine and piperazine moieties into $\mathrm{C}-16$ and $\mathrm{C}-19$ position through connecting fragments, and the D-ring structure was modified. The inhibitory effects on Colo-16 and A549 were observed, the results showed that the inhibitory activities of 4 compounds on Colo-16 were significantly better than the positive control of 5-fluorouracil. The inhibitory effects on E. coli 1924 , S. aureus $4220, S$. mutans 3289, MRSA 3167 and QRSA 3505 were also observed, the results showed that the minimum inhibitory concentration (MIC) of 6 compounds on $S$. mutans 3289 was $8 \mu \mathrm{g} / \mathrm{mL}$, which was comparable to the positive control chloramphenicol. The MIC of 16 $\beta$-O-(5-oxo-5-(4-methyl-1-piperazinyl)-valeryl)]-19-beyeran acid ethyl ester (IS-12b) on MRSA 3167 was $8 \mu \mathrm{g} / \mathrm{mL}$, which was comparable to the positive control chloramphenicol.
\end{abstract}

Keywords isosteviol; cytotoxic activity; antibacterial activity

* Corresponding authors. E-mail: 1xzhao@Innu.edu.cn; piaohua88@163.com

Received May 13, 2019; revised June 26, 2019; published online August 1, 2019.

Project supported by the National Natural Science Foundation of China (No. 21101067).

国家自然科学基金(No. 21101067)资助项目. 
异甜菊醇(Isosteviol，IS)又称异斯特维醇(图 1), 其 骨架由甜菊苷酸水解而成. 异甜菊醇是一种具有贝叶烷 骨架的四环二萜类化合物, 具有广泛的生物活性如抗肿 瘤 $^{[1 \sim 4]}$ 、抑菌 ${ }^{[5]}$ 、降血压 ${ }^{[6]}$ 、降血糖 ${ }^{[7]}$ 及抗氧化等 ${ }^{[8 \sim 10]}$.
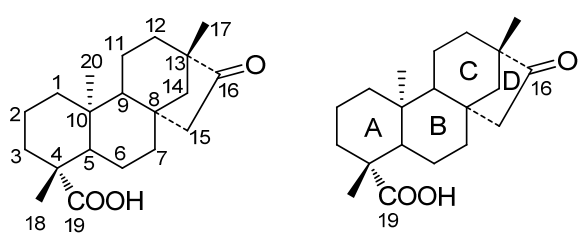

图 1 异甜菊醇的结构

Figure 1 Structure of isosteviol

通过对甜菊苷的代谢产物甜菊醇和水解产物异甜 菊醇的研究发现, 异甜菊醇比母体甜菊苷和同分异构体 甜菊醇具有更广泛的生物活性. 此外, 由于其独特而稀 有的四环二萜类结构特点使其成为药物合成中有用的
活性框架 ${ }^{[11]}$. 异甜菊醇分子中结构修饰较多的部分是 $\mathrm{D}$ 环中的酮基, 其次是 C-19 位的羧基, 成为良好的结构修 饰位点 ${ }^{[12]}$.

含氮片段的导入不仅能增加化合物的极性，同时还 可通过氢键与多种受体部位结合. 吗啉、哌嗪和 $N$-甲基 哌嗪是常见的含氮六元杂环. 许多抗肿瘤药物和抗菌药 物，如达沙替尼、波舒替尼、泊那替尼、吉非替尼、洛 美沙星和环丙沙星等, 他们的结构中都含有哌嗪或吗啉 片段. 本课题组前期的研究结果表明 ${ }^{[13 ~ 15]}$, 在五环三萜 类化合物中引入哌嗪或吗啉片段能抑制人肺腺癌细胞 (A549)等癌细胞在体外细胞系中的生长. 因此, 我们将 哌嗪、甲基哌嗪和吗啉片段导入到异甜菊醇结构中，同 时对其 D 环进行结构改造，以期得到活性较好且生物利 用度较高的新型四环二萜类衍生物. 具体合成路线如 Schemes $1 \sim 4$.

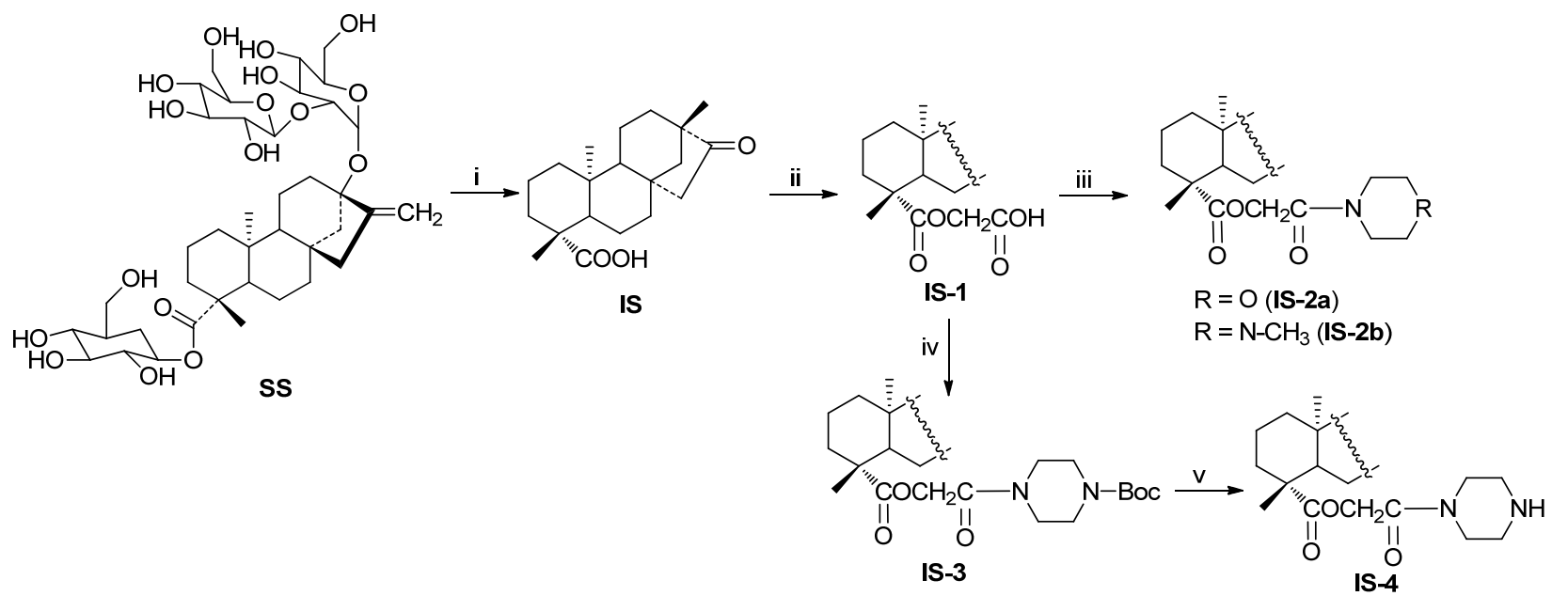

Reagents and conditions: (i) $\mathrm{H}_{2} \mathrm{SO}_{4}(w=10 \%), 95{ }^{\circ} \mathrm{C}$; (ii) $(\mathrm{COCl})_{2}, \mathrm{CH}_{2} \mathrm{Cl}_{2}$, r.t.; $\mathrm{HOCH}_{2} \mathrm{COOH}$, TEA, THF, r.t.; (iii) morpholine or $N$-methyl piperazine, DMAP, EDCl, $\mathrm{CH}_{2} \mathrm{Cl}_{2}, 0{ }^{\circ} \mathrm{C} \sim$ r.t.; (iv) $\mathrm{N}$-Boc piperazine, DMAP, $\mathrm{EDCl}, \mathrm{CH}_{2} \mathrm{Cl}_{2}, 0{ }^{\circ} \mathrm{C} \sim$ r.t.; (v) TFA, $\mathrm{CH}_{2} \mathrm{Cl}_{2}, 0{ }^{\circ} \mathrm{C} \sim$ r.t.

图式 1 异甜菊醇衍生物 IS-1 IS-4 的合成

Scheme 1 Synthesis of isosteviol derivatives IS-1 $\sim$ IS-4

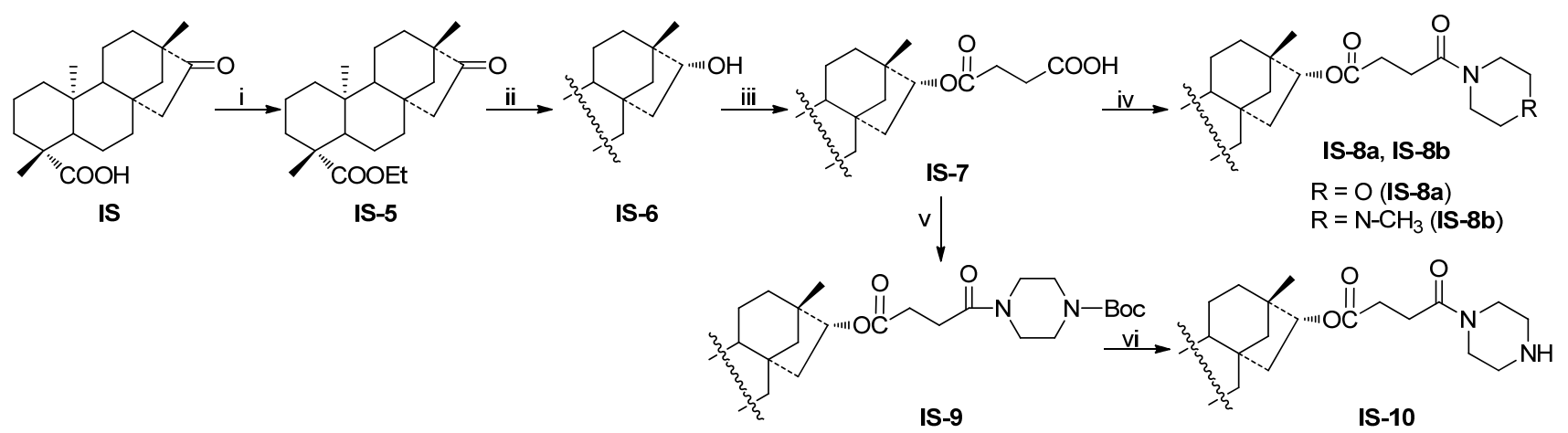

Reagents and conditions: (i) $\mathrm{CH}_{3} \mathrm{CH}_{2} \mathrm{Br}$, DMSO, $\mathrm{KOH}$, r.t.; (ii) $\mathrm{NaBH}_{4}, \mathrm{CH}_{3} \mathrm{CH}_{2} \mathrm{OH}$, r.t.; (iii) succinic anhydride, DMAP, $\mathrm{CH}_{2} \mathrm{Cl}_{2}$; (iv) morpholine or $\mathrm{N}$-methyl piperazine, DMAP, $\mathrm{EDCl}, \mathrm{CH}_{2} \mathrm{Cl}_{2}, 0{ }^{\circ} \mathrm{C} \sim$ r.t.; (v) $\mathrm{N}$-Boc piperazine, DMAP, $\mathrm{EDCl}, \mathrm{CH}_{2} \mathrm{Cl}_{2}, 0{ }^{\circ} \mathrm{C} \sim$ r.t.; $\left(\right.$ vi) $\mathrm{TFA} \mathrm{CH}_{2} \mathrm{Cl}_{2}, 0{ }^{\circ} \mathrm{C} \sim$ r.t.

图式 2 异甜菊醇衍生物 IS-7 IS-10 的合成

Scheme 2 Synthesis of isosteviol derivatives IS-7 $\sim$ IS-10 


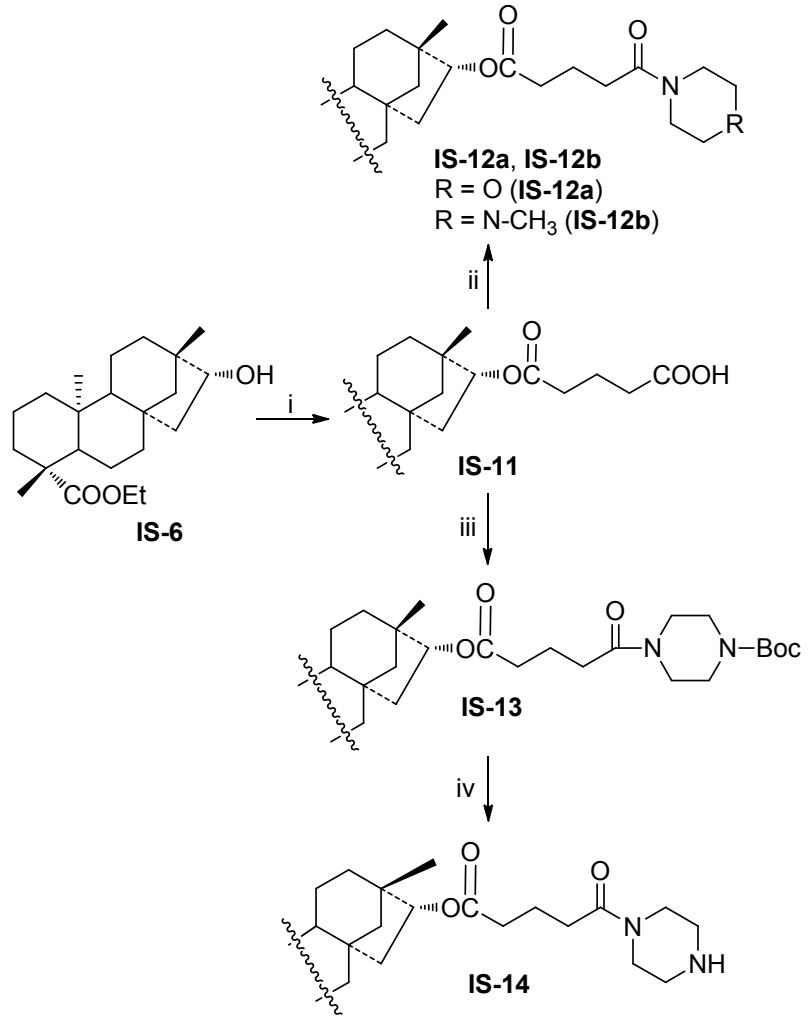

Reagents and conditions: (i) glutaric anhydride, DMAP, $\mathrm{CH}_{2} \mathrm{Cl}_{2}$; (ii) morpholine or $\mathrm{N}$-methyl piperazine, DMAP, EDCl, $\mathrm{CH}_{2} \mathrm{Cl}_{2}, 0{ }^{\circ} \mathrm{C} \sim$ r.t.; (iii) $\mathrm{N}$-Boc piperazine, DMAP, $\mathrm{EDCl}, \mathrm{CH}_{2} \mathrm{Cl}_{2}, 0{ }^{\circ} \mathrm{C} \sim$ r.t.; (iv) TFA, $\mathrm{CH}_{2} \mathrm{Cl}_{2}, 0{ }^{\circ} \mathrm{C} \sim$ r.t.

图式 3 异甜菊醇衍生物 IS-11 IS-14 的合成 Scheme 3 Synthesis of isosteviol derivatives IS-11 $\sim$ IS-14
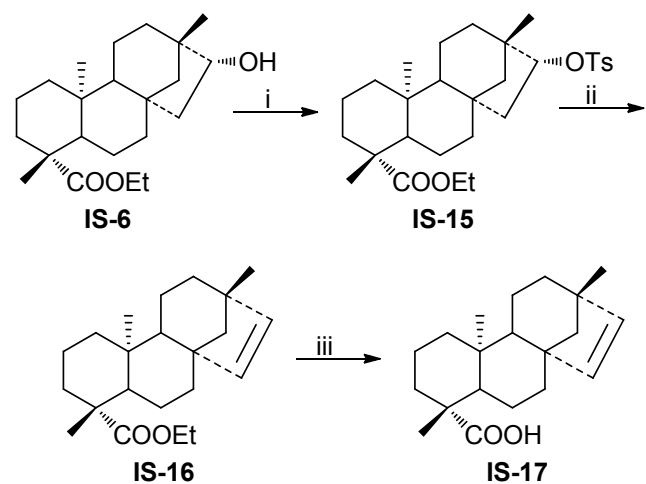

Reagents and conditions: (i) $\mathrm{TsCl}$, pyridine, r.t.; (ii) 2,4,6-collidine, reflux; (iii) Lil, 2,4,6-collidine, reflux.

图式 4 异甜菊醇衍生物 IS-16 IS-17 的合成

Scheme 4 Synthesis of isosteviol derivatives IS-16 $\sim$ IS-17

\section{1 结果与讨论}

\section{1 目标化合物的合成}

以异甜菊醇为先导化合物, 在其 C-16 位和 C-19 位 通过乙醇酸、丁二酸和戍二酸连接片段导入吗啉和哌嗪, 并对其 D 环进行结构改造, 共设计合成了 15 种未见文 献报道的异甜菊醇衍生物, 所有的新化合物结构均经
IR, ${ }^{1} \mathrm{H}$ NMR 和 ${ }^{13} \mathrm{C} \mathrm{NMR}$ 得到了确证. 同时对所有新化 合物进行了 HRMS 测试, 测得的数值与理论值相符.

\section{2 抗肿瘤细胞增殖实验}

对合成的 17 种异甜菊醇衍生物进行了细胞毒活性 测试(表 1). 根据表 1 可看出：在抑制 Colo-16 细胞测试 中，化合物 IS-1, IS-2a, IS-2b, IS-3, IS-4, IS-8a, IS-12a, IS-12b 和 IS-14 的活性优于先导化合物异甜菊醇, 其中 化合物 IS-2a, IS-4 和 IS-8a 的活性略优于阳性对照物 5氟尿嘧啶, IS-1 的活性明显优于阳性对照物 5-氟尿嘧啶; 在抑制 A549 细胞测试中，化合物 IS-9, IS-11, IS-12a, IS-16 和 IS-17 对 A549 表现出一定的抑制活性. 对具有 抗肿瘤细胞增殖活性的化合物进行构效关系分析，初步 得到以下结论: (1)在 C-19 位导入极性乙醇酸片段能明 显改善对 Colo-16 细胞的抑制; (2)通过在 C-19 位导入乙 醇酸连接片段, 与含氮六元杂环吗啉、哌嗪结构偶联的 衍生物和通过 C-19 位加入丁二酸连接片段与吗啉偶联 的衍生物均改善了对 Colo-16 细胞的抑制; (3)通过 C-19 位导入乙醇酸连接片段并与 $N$-甲基哌嗪、 $N$-Boc 哌嗪结 构偶联的衍生物和通过 C-16 位导入龙二酸连接片段并 与含氮六元杂环吗啉、 $N$-甲基哌嗪结构偶联的衍生物均 能改善对 A549 细胞的抑制活性; (4) D 环的 C-15 和 C-16 位之间变成双键时能改善对 A549 的抑制活性.

表 1 异甜菊醇衍生物抑制 Colo-16 和 A549 细胞的 $\mathrm{IC}_{50}$ 值 ${ }^{a}$ Table $1 \mathrm{IC}_{50}$ of isosteviol derivatives on Colo-16 and A549

\begin{tabular}{|c|c|c|}
\hline \multirow{2}{*}{ Compd. } & \multicolumn{2}{|c|}{$\mathrm{IC}_{50} /\left(\mu \mathrm{mol} \cdot \mathrm{L}^{-1}\right)$} \\
\hline & Colo- 16 & A549 \\
\hline IS-1 & $53.03 \pm 0.0343$ & $192.77 \pm 0.0338$ \\
\hline IS-2a & $103.37 \pm 0.1329$ & $218.62 \pm 0.0143$ \\
\hline IS-2b & $195.97 \pm 0.1057$ & $213.62 \pm 0.0335$ \\
\hline IS-3 & $190.35 \pm 0.1019$ & $200.87 \pm 0.0650$ \\
\hline IS-4 & $110.62 \pm 0.1398$ & $191.20 \pm 0.0230$ \\
\hline IS-7 & - & $224.45 \pm 0.0154$ \\
\hline IS-8a & $107.67 \pm 0.2093$ & $212.34 \pm 0.0185$ \\
\hline IS-8b & - & $242.06 \pm 0.0307$ \\
\hline IS-9 & - & $155.57 \pm 0.0273$ \\
\hline IS-10 & - & $184.00 \pm 0.0264$ \\
\hline IS-11 & $209.74 \pm 0.0842$ & $167.95 \pm 0.0415$ \\
\hline IS-12a & $192.25 \pm 0.0681$ & $145.04 \pm 0.0528$ \\
\hline IS-12b & $168.55 \pm 0.1258$ & $183.61 \pm 0.0201$ \\
\hline IS-13 & - & $183.74 \pm 0.0294$ \\
\hline IS-14 & $162.18 \pm 0.1515$ & $281.52 \pm 0.0755$ \\
\hline IS-16 & - & $165.73 \pm 0.0374$ \\
\hline IS-17 & - & $155.11 \pm 0.0800$ \\
\hline 甜菊苷(SS) & $188.41 \pm 0.127$ & $269.09 \pm 0.0659$ \\
\hline 异甜菊醇(IS) & $207.91 \pm 0.1478$ & $177.07 \pm 0.0199$ \\
\hline 齐墩果酸(OA) & - & $170.88 \pm 0.0303$ \\
\hline 阿霉素(Adriamycin) & $30.82 \pm 0.3025$ & $47.63 \pm 0.0811$ \\
\hline 5-氟尿嘧啶(5-FU) & $117.41 \pm 0.2246$ & $58.65 \pm 0.1042$ \\
\hline
\end{tabular}




\section{3 抑菌活性实验}

对所合成的异甜菊醇衍生物进行了大肠杆菌 ( $E$. coli 1924)、金黄色葡萄球菌(S. aureus 4220)、变形链球 菌(S. mutans 3289)、耐甲氧西林金黄色葡萄球菌( MRSA 3167 ) 和耐喹诺酮金黄色葡萄球菌 ( QRSA 3505) 5 种菌株 的抑菌活性测试. 在对 E. coli 1924 的抑菌活性测试中, 化合物 IS-4, IS-7, IS-8b 和 IS-11 均显示出一定的抑菌活 性; 在对 S. aureus 4220 的抑菌活性测试中, 化合物 IS-4, IS-7, IS-8b, IS-11 和 IS-12b 显示出一定的抑菌活 性; 在对 S. mutans 3289 的活性测试中, 衍生物 IS-9, IS-10, IS-12a, IS-12b, IS-14 和 IS-17 与阳性对照物氯霉 素活性相当, 对 S. mutans 3289 的 MIC 为 $8 \mu \mathrm{g} / \mathrm{mL}$; 在对 MRSA 3167 的活性测试中, 衍生物 IS-7, IS-10, IS-11, IS-12b 和 IS-17 显示出一定活性, 其中 IS-12b 最小抑菌 浓度(MIC)为 $8 \mu \mathrm{g} / \mathrm{mL}$, 与阳性对照物氯霉素相当; 在对 QRSA 3505 的活性测试中, 衍生物 IS-10, IS-11, IS-12b, IS-14 和 IS-17 显示出的活性弱于阳性对照物氯霉素. 对 具有抑菌活性的化合物进行构效关系分析, 初步得到以 下结论: (1)该类化合物对 S. mutans 3289 和 MRSA 3167 显示出较好的选择抑制性; (2) 通过丁二酸片段与 $N$-Boc 哌嗪哌嗪结构偶联, 或通过戊二酸片段与吗啉、 $N$-甲基 哌嗪或哌嗪结构偶联, 以及 D 环 C-15、C-16 位变成双 键能明显改善先导化合物对 S. mutans 3289 的抑制活性;

表 2 抗菌实验数据 ${ }^{a}$

Table 2 Anti-bacterial experimental results

\begin{tabular}{|c|c|c|c|c|c|}
\hline \multirow[b]{2}{*}{ Compd. } & \multicolumn{5}{|c|}{$\mathrm{MIC} /\left(\mu \mathrm{g} \cdot \mathrm{mL}^{-1}\right)$} \\
\hline & $\begin{array}{c}\text { E. coli } \\
1924\end{array}$ & $\begin{array}{c}\text { S. aureus } \\
4220\end{array}$ & $\begin{array}{c}\text { S. mutans } \\
3289\end{array}$ & $\begin{array}{c}M R S A \\
3167\end{array}$ & $\begin{array}{l}\text { QRSA } \\
3505\end{array}$ \\
\hline IS-1 & - & - & - & - & - \\
\hline IS-2a & $>128$ & $>128$ & 64 & - & - \\
\hline IS-2b & $>128$ & $>128$ & 128 & - & - \\
\hline IS-3 & $>128$ & $>128$ & 16 & - & - \\
\hline IS-4 & 128 & 64 & 128 & 128 & $>128$ \\
\hline IS-7 & 64 & 64 & 16 & 64 & 128 \\
\hline IS-8a & - & - & - & - & - \\
\hline IS-8b & 16 & 16 & $>128$ & 128 & $>128$ \\
\hline IS-9 & $>128$ & $>128$ & 8 & - & - \\
\hline IS-10 & $>128$ & $>128$ & 8 & 16 & 16 \\
\hline IS-11 & 32 & 32 & 32 & 64 & 64 \\
\hline IS-12a & $>128$ & $>128$ & 8 & - & - \\
\hline IS-12b & $>128$ & 32 & 8 & 8 & 32 \\
\hline IS-13 & - & - & - & - & - \\
\hline IS-14 & $>128$ & $>128$ & 8 & $>128$ & 16 \\
\hline IS-16 & - & - & - & - & - \\
\hline IS-17 & $>128$ & $>128$ & 8 & 32 & 16 \\
\hline Stevioside (SS) & - & - & - & - & - \\
\hline Isosteviol (IS) & - & - & - & - & - \\
\hline Chloramphenicol & 4 & 4 & 8 & 8 & 8 \\
\hline
\end{tabular}

a "_" Indicates that the compound has no inhibitory effect on the tested bacteria..
(3)通过丁二酸片段与哌嗪结构偶联, 或通过戊二酸片 段与 $N$-甲基哌嗪结构偶联, 以及 $\mathrm{D}$ 环 C- $15, \mathrm{C}-16$ 位变成 双键能改善先导化合物对 MRSA 3167 的抑制活性; (4) 通过丁二酸片段与哌嗪结构偶联, 或通过戊二酸片段与 $N$-甲基哌嗪、哌嗪结构偶联，以及 D 环 C- $15, \mathrm{C}-16$ 位变 成双键能改善先导化合物对 $Q R S A 3505$ 的抑制活性.

\section{2 结论}

以异甜菊醇为先导化合物, 通过连接片段分别在 C-16 和 C-19 位引入吗啉和哌嗪结构, 并对 D 环进行结 构改造，设计合成了 15 种未见文献报道的异甜菊醇衍 生物, 并观察其对人皮肤鳞癌细胞(Colo-16)和人肺腺癌 细胞(A549)抑制作用. 通过活性对比发现, 导入乙醇酸 连接片段能明显改善对 Colo-16 细胞的抑制; 通过在 C-19 位导入乙醇酸连接片段与含氮六元杂环吗啉、哌嗪 结构偶联的衍生物和通过 C-19 位加入丁二酸连接片段 与吗啉偶联的衍生物均改善了对 Colo-16 细胞的抑制; 通过 $\mathrm{C}-19$ 位导入乙醇酸连接片段并与 $N$-甲基哌嗪、 $N$-Boc 哌嗪结构偶联的衍生物和通过 C-16 位导入戊二 酸连接片段并与含氮六元杂环吗啉、 $N$-甲基哌嗪结构偶 联的衍生物均能改善对 A549 细胞的抑制活性; D 环的 C-15 和 C-16 位之间变成双键时能改善对 A549 的抑制 活性. 通过丁二酸片段与 $N$-Boc 哌嗪哌嗪结构偶联, 或 通过戊二酸片段与吗啉、 $N$-甲基哌嗪或哌嗪结构偶联, 以及 D 环 C-15、C-16 位变成双键能明显改善先导化合 物对 S. mutans 3289 的抑制活性; 通过丁二酸片段与哌 嗪结构偶联, 或通过戊二酸片段与 $N$-甲基哌嗪结构偶 联，以及 D 环 C-15、C-16 位变成双键能改善先导化合 物对 MRSA 3167 的抑制活性; 通过丁二酸片段与哌嗪 结构偶联, 或通过戊二酸片段与 $N$-甲基哌嗪、哌嗪结构 偶联，以及 D 环 C-15, C-16 位变成双键能改善先导化合 物对 $Q R S A 3505$ 的抑制活性. 以上结果为以后异甜菊醇 的结构修饰改造提供了方向.

\section{3 实验部分}

\section{1 仪器与试剂}

X-5 型纤维熔点测定仪(温控型)(北京泰克仪器有限 公司); WGH-30 型双光束红外分光光度计(上海勇规分 析仪器有限公司), KBr 压片; VARIAN MRTCURY PLUS 400 型核磁共振仪 (美国 Varian 技术有限公司), AVANCE-500 MHz 核磁共振仪(瑞士 BRUKER 公司), $\mathrm{CDCl}_{3}$ 为溶剂; $\mathrm{GC}$-Tof $\mathrm{MS}$ 型高分辨率质谱. 所用药品 和试剂均为分析纯或化学纯.

\section{2 实验方法}

16-氧亚基-贝叶-19-酸(IS)的合成参考文献[16], 得 
到白色固体产物. 16-氧亚基-贝叶-19-酸乙酯的具体合 成方法见参考文献[17], 得到了白色的固体产物 IS-5. m.p. $126 \sim 127{ }^{\circ} \mathrm{C} .16 \beta$-差基-贝叶-19-酸乙酯的具体合 成方法见参考文献[17], 得到了白色的固体产物 IS-6. m.p. $152 \sim 153{ }^{\circ} \mathrm{C}$; 16 $\beta$ - $O$-[4-氧亚基-4-甲苯磺酰基]-贝 叶-19-酸乙酯的合成参考文献[18], 得到无色液体产物 IS-15.

3.2.12-(16-氧亚基-贝叶-19-氧亚基-19-氧)乙酸 (IS-1) 的合成

在圆底烧瓶中加入二氯甲烷 $(15 \mathrm{~mL})$, 将 IS (1.0g, $14.00 \mathrm{~mol})$ 溶于其中, 在搅拌下加入草酰氯(1.1 mL, $12.56 \mathrm{mmol}$ ), 常温下搅拌 $24 \mathrm{~h}$, 薄层层析(TLC)检测反 应, 结束后, 减压蒸馏除去溶剂, 再加入乙醇酸(477.6 $\mathrm{mg}, 6.28 \mathrm{mmol})$ 和三乙胺 $(4.4 \mathrm{~mL}, 31.40 \mathrm{mmol})$, 在搅拌 下加入四氢呋喃, 室温下反应 $7 \mathrm{~h}$, 反应完成后, 减压蒸 馏除去溶剂, 用乙酸乙酯和水萃取, 并用 $1 \mathrm{~mol} / \mathrm{L} \mathrm{HCl}$ 洗涤至微酸性, 用饱和氯化钠洗涤, 过滤, 柱层析得 $490.2 \mathrm{mg}$ 白色固体产物 IS-1, 产率 42\%. m.p. 188.5 $188.9{ }^{\circ} \mathrm{C} ;[\alpha]_{\mathrm{D}}^{16}-59.1\left(c 0.04, \mathrm{CH}_{2} \mathrm{Cl}_{2}\right) ;{ }^{1} \mathrm{H} \mathrm{NMR}\left(\mathrm{CDCl}_{3}\right.$, $500 \mathrm{MHz}) \delta: 6.92(\mathrm{~s}, 1 \mathrm{H}, \mathrm{COOH}), 4.67(\mathrm{~d}, J=16.3 \mathrm{~Hz}$, 1H, OCHCO), 4.58 (d, $J=16.3 \mathrm{~Hz}, 1 \mathrm{H}, \mathrm{OCHCO}), 2.64$ $\left(\mathrm{dd}, J=18.7,3.0 \mathrm{~Hz}, 1 \mathrm{H}, \mathrm{H}_{\alpha}-15\right), 2.24$ (d, $J=13.4 \mathrm{~Hz}, 1 \mathrm{H}$, $\mathrm{H}_{\beta^{-}}$15), 1.90 (d, $\left.J=13.6 \mathrm{~Hz}, 1 \mathrm{H}\right), 1.80$ (d, $J=19.9 \mathrm{~Hz}$, 2H), $1.76 \sim 1.65(\mathrm{~m}, 4 \mathrm{H}), 1.60(\mathrm{~d}, J=11.8 \mathrm{~Hz}, 1 \mathrm{H}), 1.55$ $(\mathrm{d}, J=11.9 \mathrm{~Hz}, 1 \mathrm{H}), 1.51 \sim 1.34(\mathrm{~m}, 4 \mathrm{H}), 1.26(\mathrm{~s}, 3 \mathrm{H})$, $1.24 \sim 1.15(\mathrm{~m}, 3 \mathrm{H}), 1.09 \sim 1.03(\mathrm{~m}, 1 \mathrm{H}), 0.97(\mathrm{~s}, 3 \mathrm{H})$, $0.94 \sim 0.88(\mathrm{~m}, 1 \mathrm{H}), 0.73(\mathrm{~s}, 3 \mathrm{H}) ;{ }^{13} \mathrm{C} \mathrm{NMR}\left(\mathrm{CDCl}_{3}, 125\right.$ $\mathrm{MHz}) \delta: 223.3,176.9,173.0,60.2,57.3,54.9,54.5,49.0$, $48.7,44.2,41.7,40.0,39.7,38.3,38.1,37.5,29.1,21.8$, 20.5, 20.1, 19.1, 13.7; IR (KBr) v: 3447, 2941, 2845, 1740, 1462, $1148 \mathrm{~cm}^{-1}$; HRMS calcd for $\mathrm{C}_{22} \mathrm{H}_{32} \mathrm{O}_{5} 376.2250$, found 376.2248 .

3.2 .2 (16-氧亚基-贝叶-19)-酸-[2-(4-吗啉基)-2-氧亚 基]乙酯(IS-2a) 的合成

在圆底烧瓶中加入二氯甲烷 $(5 \mathrm{~mL})$, 依次将 IS-1 (100.0 mg, $0.26 \mathrm{mmol}) 、$ 吗啉 $(0.03 \mathrm{~mL}, 0.31 \mathrm{mmol})$ 和 4二甲氨基吡定(DMAP) $(38.1 \mathrm{mg}, 0.31 \mathrm{mmol})$ 溶于其中, 搅拌下将反应液冷却到 $0{ }^{\circ} \mathrm{C}$. 再将二亚胺盐酸盐 (EDCI) (249.2 mg, $1.30 \mathrm{mmol}$ )溶于二氯甲烷中，逐滴将 其加入反应液中, 于 $0{ }^{\circ} \mathrm{C}$ 下摚拌 $5 \mathrm{~min}$, 然后室温下摚 拌 $3 \mathrm{~h}$. 薄层层析检测反应结束后, 减压蒸馏除去溶剂, 用二氯甲烷和水萃取, 并用饱和氯化钠溶液洗涤至中 性, 用无水硫酸镁对有机相进行干燥, 过滤, 柱层析得 $104.2 \mathrm{mg}$ 白色固体 IS-2a, 产率 88\%. m.p. 55.1 $55.7{ }^{\circ} \mathrm{C} ; \quad[\alpha]_{\mathrm{D}}^{20}-53.5\left(c \quad 0.40, \mathrm{CH}_{2} \mathrm{Cl}_{2}\right) ;{ }^{1} \mathrm{H} \quad \mathrm{NMR}$
$\left(\mathrm{CDCl}_{3}, 500 \mathrm{MHz}\right) \delta: 4.80(\mathrm{~d}, J=14.3 \mathrm{~Hz}, 1 \mathrm{H}, \mathrm{OCHCO})$, $4.63(\mathrm{~d}, J=14.3 \mathrm{~Hz}, 1 \mathrm{H}, \mathrm{OCHCO}), 3.71 \sim 3.42(\mathrm{~m}, 8 \mathrm{H}$, morpholine $\mathrm{H}-2 \times 2, \mathrm{H}-3 \times 2, \mathrm{H}-5 \times 2, \mathrm{H}-6 \times 2), 2.65$ (dd, $\left.J=18.7,3.4 \mathrm{~Hz}, 1 \mathrm{H}, \mathrm{H}_{\alpha}-15\right), 2.28(\mathrm{~d}, J=13.5 \mathrm{~Hz}, 1 \mathrm{H}$, $\left.\mathrm{H}_{\beta^{-}}-15\right), 1.94(\mathrm{~d}, J=13.9 \mathrm{~Hz}, 1 \mathrm{H}), 1.87 \sim 1.78(\mathrm{~m}, 3 \mathrm{H})$, $1.73(\mathrm{~d}, J=14.5 \mathrm{~Hz}, 1 \mathrm{H}), 1.68(\mathrm{~d}, J=13.9 \mathrm{~Hz}, 1 \mathrm{H}), 1.63 \sim$ $1.52(\mathrm{~m}, 4 \mathrm{H}), 1.50 \sim 1.41(\mathrm{~m}, 3 \mathrm{H}), 1.32(\mathrm{~s}, 3 \mathrm{H}), 1.23 \sim$ $1.17(\mathrm{~m}, 3 \mathrm{H}), 1.11 \sim 1.05(\mathrm{~m}, 1 \mathrm{H}), 0.99(\mathrm{~s}, 3 \mathrm{H}), 0.96 \sim$ $0.90(\mathrm{~m}, 1 \mathrm{H}), 0.75(\mathrm{~s}, 3 \mathrm{H}) ;{ }^{13} \mathrm{C} \mathrm{NMR}\left(\mathrm{CDCl}_{3}, 125 \mathrm{MHz}\right) \delta$ : 222.8, 177.1, 165.2, 60.9, 57.3, 55.0, 54.9, 54.7, 54.5, 48.9, $48.7,46.2$, 44.6, 44.3, 41.9, 41.7, 40.0, 39.7, 38.3, 38.2, $37.5,29.3,21.9,20.5,20.1,19.2$, 13.8; IR (KBr) v: 2951, 2851, 1736, 1676, 1458, $1229 \mathrm{~cm}^{-1}$; HRMS calcd for $\mathrm{C}_{26} \mathrm{H}_{39} \mathrm{NO}_{5}$ 445.2828, found 4452817.

3.2.3 (16-氧亚基-贝叶-19)-酸-[2-(4-甲基-1-哌嗪 基)-2-氧亚基]乙酯(IS-2b)的合成

在圆底烧瓶中加入二氯甲烷 $(5 \mathrm{~mL})$, 依次将 IS-1 (80.0 mg, $0.21 \mathrm{mmol}) 、 N$-甲基哌嗪( $0.05 \mathrm{~mL}, 0.42 \mathrm{mmol})$ 和 DMAP (51.3 mg, $0.42 \mathrm{mmol}$ )溶于其中，搅拌下将反应 液冷却到 $0{ }^{\circ} \mathrm{C}$. 再将 EDCI $(201.3 \mathrm{mg}, 1.05 \mathrm{mmol})$ 溶于 二氯甲烷中，逐滴将其加入反应液中，于 $0{ }^{\circ} \mathrm{C}$ 下搅拌 5 $\min$, 然后室温下搅拌 $3 \mathrm{~h}$. 薄层层析检测反应结束后, 减压蒸馏除去溶剂, 用二氯甲烷和水进行萃取, 用饱和 氯化钠溶液洗涤至中性, 无水硫酸镁干燥, 过滤, 柱层 析得到无色液体产物 IS-2b, 产率 $92.0 \%$. [ $\alpha]_{\mathrm{D}}^{21}-36.08$ (c $\left.0.19, \mathrm{CH}_{2} \mathrm{Cl}_{2}\right) ;{ }^{1} \mathrm{H} \mathrm{NMR}\left(\mathrm{CDCl}_{3}, 500 \mathrm{MHz}\right) \delta: 4.79$ (d, $J=14.2 \mathrm{~Hz}, 1 \mathrm{H}, \mathrm{OCHCO}), 4.61$ (d, $J=14.2 \mathrm{~Hz}, 1 \mathrm{H}$, OCHCO), 3.62 (s, $2 \mathrm{H}$, piperazine $\mathrm{H}-2 \times 2), 3.41$ (s, $2 \mathrm{H}$, piperazine $\mathrm{H}-6 \times 2), 2.63\left(\mathrm{dd}, J=18.7,3.4 \mathrm{~Hz}, 1 \mathrm{H}, \mathrm{H}_{\alpha^{-}}-15\right)$, $2.40(\mathrm{~d}, J=5.3 \mathrm{~Hz}, 4 \mathrm{H}$, piperazine $\mathrm{H}-3 \times 2, \mathrm{H}-5 \times 2)$, $2.30\left(\mathrm{~s}, 3 \mathrm{H}, \mathrm{NCH}_{3}\right), 2.27$ (d, $\left.J=13.7 \mathrm{~Hz}, 1 \mathrm{H}, \mathrm{H}_{\beta}-15\right), 1.93$ $(\mathrm{d}, J=13.3 \mathrm{~Hz}, 1 \mathrm{H}), 1.86 \sim 1.77(\mathrm{~m}, 3 \mathrm{H}), 1.69$ (q, $J=14.1$ $\mathrm{Hz}, 3 \mathrm{H}), 1.60$ (d, $J=11.7 \mathrm{~Hz}, 1 \mathrm{H}), 1.54(\mathrm{~d}, J=11.7 \mathrm{~Hz}$, $1 \mathrm{H}), 1.51 \sim 1.39(\mathrm{~m}, 4 \mathrm{H}), 1.30(\mathrm{~s}, 3 \mathrm{H}), 1.21 \sim 1.15(\mathrm{~m}$, $3 \mathrm{H}), 1.09 \sim 1.03(\mathrm{~m}, 1 \mathrm{H}), 0.97(\mathrm{~s}, 3 \mathrm{H}), 0.95 \sim 0.88(\mathrm{~m}$, $1 \mathrm{H}), 0.74$ (s, 3H); ${ }^{13} \mathrm{C} \mathrm{NMR}\left(\mathrm{CDCl}_{3}, 125 \mathrm{MHz}\right) \delta: 222.8$, 177.1, 165.2, 60.9, 57.3, 55.0, 54.9, 54.7, 54.5, 48.9, 48.7, 46.2, 44.6, 44.3, 41.9, 41.7, 40.0, 39.7, 38.3, 38.2, 37.5, 29.3, 21.9, 20.5, 20.1, 19.2, 13.8; IR (KBr) v: 2949, 2869, 1736, 1680, 1456, 1240, $1173 \mathrm{~cm}^{-1}$; HRMS calcd for $\mathrm{C}_{27} \mathrm{H}_{42} \mathrm{~N}_{2} \mathrm{O}_{4} 458.3145$, found 458.3149 .

3.2 .4 (16-氧亚基-贝叶-19)-酸-[2-(4-叔丁氧羰基-1哌嗪基)-2-氧亚基]乙酯(IS-3)的合成

在圆底烧瓶中加入二氯甲烷 $(5 \mathrm{~mL})$, 依次将 IS-1 (90.0 mg, $0.24 \mathrm{mmol}) 、 N$-Boc 哌嗪(88.7 mg, $0.48 \mathrm{mmol})$ 
和 DMAP (58.2 mg, $0.48 \mathrm{mmol}$ )溶于其中, 将反应液冷却 到 $0{ }^{\circ} \mathrm{C}$. 再将 EDCI $(277.9 \mathrm{mg}, 1.19 \mathrm{mmol})$ 溶于无水二 氯甲烷中, 逐滴将其加入反应液中, 于 $0{ }^{\circ} \mathrm{C}$ 下搅拌 5 $\min$, 然后室温下搅拌 $3 \mathrm{~h}$. 薄层层析检测反应结束后, 减压蒸馏除去溶剂, 用二氯甲烷和水萃取, 并用饱和氯 化钠溶液洗涤至中性, 无水硫酸镁对有机相进行干燥, 过滤, 柱层析得 $92.1 \mathrm{mg}$ 无色液体产物 IS-3, 产率 71\%. $[\alpha]_{\mathrm{D}}^{18}-39.9$ (c $\left.0.20, \mathrm{CH}_{2} \mathrm{Cl}_{2}\right) ;{ }^{1} \mathrm{H}$ NMR $\left(\mathrm{CDCl}_{3}, 500\right.$ MHz) $\delta: 4.81$ (d, $J=14.3 \mathrm{~Hz}, 1 \mathrm{H}$, OCHCO), 4.63 (d, $J=$ $14.25 \mathrm{~Hz}, 1 \mathrm{H}, \mathrm{OCHCO}), 3.58 \sim 3.38(\mathrm{~m}, 8 \mathrm{H}$, piperazine $\mathrm{H}-2 \times 2, \mathrm{H}-3 \times 2, \mathrm{H}-5 \times 2, \mathrm{H}-6 \times 2), 2.64(\mathrm{~d}, J=17.7 \mathrm{~Hz}$, $\left.1 \mathrm{H}, \mathrm{H}_{\alpha^{-}}-15\right), 2.27$ (d, $\left.J=13.3 \mathrm{~Hz}, 1 \mathrm{H}, \mathrm{H}_{\beta^{-}}-15\right), 1.93$ (d, $J=$ $13.8 \mathrm{~Hz}, 1 \mathrm{H}), 1.80(\mathrm{q}, J=18.5 \mathrm{~Hz}, 3 \mathrm{H}), 1.73 \sim 1.54(\mathrm{~m}$, 6H), 1.47 (s, 9H), 1.38 (q, $J=13.5 \mathrm{~Hz}, 3 \mathrm{H}), 1.31(\mathrm{~s}, 3 \mathrm{H})$, $1.18(\mathrm{t}, J=10.9 \mathrm{~Hz}, 3 \mathrm{H}), 1.10 \sim 1.04(\mathrm{~m}, 1 \mathrm{H}), 0.98(\mathrm{~s}, 3 \mathrm{H})$, $0.95 \sim 0.89(\mathrm{~m}, 1 \mathrm{H}), 0.74(\mathrm{~s}, 3 \mathrm{H}) ;{ }^{13} \mathrm{C} \mathrm{NMR}\left(\mathrm{CDCl}_{3}, 125\right.$ MHz) $\delta$ : 177.5, 173.4, 172.6, 172.1, 81.7, 59.9, 57.1, 57.0, 55.7, 54.8, 52.1, 43.7, 42.4, 41.5, 40.7, 39.9, 38.1, 38.1, $35.5,34.6,33.6,31.2,28.9,24.9,21.7,21.1,20.2,19.0$, 18.9, 17.8, 14.1, 13.3; IR (KBr) v: 3445, 2930, 2851, 1734, $1672,1456,1236,1166 \mathrm{~cm}^{-1}$; HRMS calcd for $\mathrm{C}_{31} \mathrm{H}_{48^{-}}$ $\mathrm{N}_{2} \mathrm{O}_{6} 544.3512$, found 544.3511 .

3.2 .5 (16-氧亚基-贝叶-19)-酸-[2-(1-哌嗪基)-2-氧亚 基]乙酯(IS-4)的合成

将 IS-3 (100.0 mg, $0.18 \mathrm{mmol}$ )溶于二氯甲烷中, 冷 却到 $0{ }^{\circ} \mathrm{C}$ 后加入三氟乙酸 $(0.4 \mathrm{~mL}, 5.40 \mathrm{mmol})$, 再将温 度升到室温搅拌 $2 \mathrm{~h}$, 反应结束后用 $0.1 \mathrm{~mol} \cdot \mathrm{L}^{-1}$ $\mathrm{NH}_{3} \cdot \mathrm{H}_{2} \mathrm{O}$ 中和至微碱性 $(\mathrm{pH}=9$, 冰水浴), 用二氯甲烷 和水萃取, 有机相用无水硫酸镁干燥, 过滤, 经柱层析 得 $61.5 \mathrm{mg}$ 无色液体产物 IS-4, 产率 75\%. [ $\alpha]_{\mathrm{D}}^{19}-39.5$ (c 0.04, $\left.\mathrm{CH}_{2} \mathrm{Cl}_{2}\right) ;{ }^{1} \mathrm{H}$ NMR $\left(\mathrm{CDCl}_{3}, 500 \mathrm{MHz}\right) \delta: 4.81(\mathrm{~d}$, $J=17.8 \mathrm{~Hz}, 1 \mathrm{H}$, OCHCO), 4.63 (d, $J=17.8 \mathrm{~Hz}, 1 \mathrm{H}$, OCHCO), $3.62 \sim 2.91(\mathrm{~m}, 8 \mathrm{H}$, piperazine $\mathrm{H}-2 \times 2, \mathrm{H}-3 \times 2$, $\mathrm{H}-5 \times 2 \& \mathrm{H}-6 \times 2$ ), 2.65 (dd, $J=23.3,4.6 \mathrm{~Hz}, 1 \mathrm{H}, \mathrm{H}_{\alpha}-15$ ), 2.29 (d, $\left.J=16.8 \mathrm{~Hz}, 1 \mathrm{H}, \mathrm{H}_{\beta^{-}}-15\right), 1.95(\mathrm{~d}, J=17.5 \mathrm{~Hz}, 1 \mathrm{H})$, $1.85 \sim 1.78(\mathrm{~m}, 3 \mathrm{H}), 1.75 \sim 1.66(\mathrm{~m}, 4 \mathrm{H}), 1.57$ (q, $J=11.7$ $\mathrm{Hz}, 1 \mathrm{H}), 1.43$ (q, $J=10.2 \mathrm{~Hz}, 2 \mathrm{H}), 1.32(\mathrm{~s}, 3 \mathrm{H}), 1.27$ (s, $3 \mathrm{H}), 1.12 \sim 1.04(\mathrm{~m}, 2 \mathrm{H}), 0.99(\mathrm{~s}, 3 \mathrm{H}), 0.95$ (q, $J=11.7$ $\mathrm{Hz}, 1 \mathrm{H}), 0.76(\mathrm{~s}, 3 \mathrm{H}) ;{ }^{13} \mathrm{C} \mathrm{NMR}\left(\mathrm{CDCl}_{3}, 125 \mathrm{MHz}\right) \delta$ : 176.9, 165.3, 154.5, 80.5, 60.7, 57.1, 54.7, 54.3, 48.7, 48.5, $44.1,41.5,41.0,39.8,39.5,38.1,38.0,37.3,29.0,28.4$ (2C), 21.6, 20.3, 19.9, 19.0, 13.6; IR (KBr) v: 3451, 2928, 2851, 1734, 1670,1458, $1153 \mathrm{~cm}^{-1}$; HRMS calcd for $\mathrm{C}_{26} \mathrm{H}_{40}-\mathrm{N}_{2} \mathrm{O}_{4} 444.2988$, found 444.2985 .
3.2.64 4-氧亚基-4-(16 $\beta-O-19$-氧亚基-19-乙氧基-贝 叶)丁酸(IS-7)的合成

在圆底烧瓶中加入二氯甲烷 $(5 \mathrm{~mL})$, 依次将 IS-6 $(100.0 \mathrm{mg}, 0.29 \mathrm{mmol}) 、 丁 二$ 酸酎 $(57.4 \mathrm{mg}, 0.57 \mathrm{mmol})$ 和 DMAP (42.1 mg, $0.35 \mathrm{mmol}$ )溶于其中, 加热回流 $8 \mathrm{~h}$, 薄层层析检测反应结束后, 用二氯甲烷和水萃取, 有机 相用无水硫酸镁干燥, 过滤, 经柱层析得 $80.1 \mathrm{mg}$ 无色 液体 IS-7, 产率 63\%. $[\alpha]_{\mathrm{D}}^{21}-57.7\left(c 0.18, \mathrm{CH}_{2} \mathrm{Cl}_{2}\right) ;{ }^{1} \mathrm{H}$ NMR $\left(\mathrm{CDCl}_{3}, 400 \mathrm{MHz}\right) \delta: 4.76 \sim 4.73(\mathrm{~m}, 1 \mathrm{H}, \mathrm{CHOCO})$, $4.15 \sim 4.00\left(\mathrm{~m}, 2 \mathrm{H}, \mathrm{OCH}_{2} \mathrm{CH}_{3}\right), 2.69 \sim 2.62(\mathrm{~m}, 4 \mathrm{H}$, $\left.\mathrm{OCH}_{2} \mathrm{CH}_{2} \mathrm{CO}\right), 2.15$ (d, $\left.J=13.2 \mathrm{~Hz}, 1 \mathrm{H}, \mathrm{H}_{\alpha}-15\right), 1.88 \sim$ $1.69(\mathrm{~m}, 6 \mathrm{H}), 1.60 \sim 1.46(\mathrm{~m}, 4 \mathrm{H}), 1.44 \sim 1.32(\mathrm{~m}, 3 \mathrm{H})$, $1.25(\mathrm{t}, J=7.2 \mathrm{~Hz}, 4 \mathrm{H}), 1.15(\mathrm{~s}, 3 \mathrm{H}), 1.07 \sim 0.94(\mathrm{~m}, 4 \mathrm{H})$, 0.90 (s, 3H), 0.85 (q, $J=9.6 \mathrm{~Hz}, 1 \mathrm{H}), 0.70(\mathrm{~s}, 3 \mathrm{H}) ;{ }^{13} \mathrm{C}$ $\mathrm{NMR}\left(\mathrm{CDCl}_{3}, 100 \mathrm{MHz}\right) \delta: 178.2,177.8,172.4,138.1$, 82.4, 60.2, 57.3, 55.9, 55.0, 43.9, 42.6, 41.8, 41.7, 40.7, $40.1,38.3,34.8,29.4,29.3,25.1,21.9,20.4,19.1,14.4$, 13.4; IR (KBr) v: 3437, 2947, 2846, 1719, 1452, 1379, $1229,1177 \mathrm{~cm}^{-1}$; HRMS calcd for $\mathrm{C}_{26} \mathrm{H}_{40} \mathrm{NO}_{6} 448.2825$, found 448.2855 .

3.2.7 16 3 - $O$-(4-氧亚基-4-吗啉基-丁酰基)-19-贝叶 酸乙酯(IS-8a)的合成

按照合成 IS-2a 的方法由化合物 IS-7 (98.0 mg, 0.22 $\mathrm{mmol}) 、$ 吗啉 $(0.02 \mathrm{~mL}, 0.26 \mathrm{mmol}) 、 \mathrm{DMAP}(32.0 \mathrm{mg}, 0.26$ $\mathrm{mmol}$ )和 EDCI (209.00 mg, $1.09 \mathrm{mmol}$ )反应, 得 $87.0 \mathrm{mg}$ 白色固体产物 IS-8a, 产率 75\%. m.p. 99.1 99.7 ${ }^{\circ} \mathrm{C}$. $[\alpha]_{\mathrm{D}}^{16}-45.3\left(c \quad 0.38, \mathrm{CH}_{2} \mathrm{Cl}_{2}\right) ;{ }^{1} \mathrm{H}$ NMR $\left(\mathrm{CDCl}_{3}, 500\right.$ MHz) $\delta: 4.75 \sim 4.71(\mathrm{~m}, 1 \mathrm{H}, \mathrm{CHOCO}), 4.14 \sim 3.99(\mathrm{~m}$, $\left.2 \mathrm{H}, \mathrm{OCH}_{2} \mathrm{CH}_{3}\right), 3.69 \sim 3.45(\mathrm{~m}, 8 \mathrm{H}$, morpholine $\mathrm{H}-2 \times 2$, $\mathrm{H}-3 \times 2, \mathrm{H}-5 \times 2, \mathrm{H}-6 \times 2), 2.71 \sim 2.59\left(\mathrm{~m}, 4 \mathrm{H}, \mathrm{COCH}_{2}-\right.$ $\mathrm{CH}_{2} \mathrm{CO}$ ), $2.15\left(\mathrm{~d}, J=16.8 \mathrm{~Hz}, 1 \mathrm{H}, \mathrm{H}_{\alpha}-15\right), 1.83 \sim 1.76(\mathrm{~m}$, $5 \mathrm{H}), 1.70(\mathrm{~d}, J=16.1 \mathrm{~Hz}, 1 \mathrm{H}), 1.61(\mathrm{~s}, 3 \mathrm{H}), 1.58 \sim 1.49$ $(\mathrm{m}, 4 \mathrm{H}), 1.41 \sim 1.31(\mathrm{~m}, 3 \mathrm{H}), 1.20(\mathrm{t}, J=9.2 \mathrm{~Hz}, 3 \mathrm{H}), 1.15$ (s, 3H), 1.00 (d, $J=5.1 \mathrm{~Hz}, 1 \mathrm{H}), 0.90$ (s, 3H), 0.85 (q, $J=$ $11.6 \mathrm{~Hz}, 1 \mathrm{H}), 0.71(\mathrm{~s}, 3 \mathrm{H}) ;{ }^{13} \mathrm{C} \mathrm{NMR}\left(\mathrm{CDCl}_{3}, 125 \mathrm{MHz}\right) \delta$ : $177.8,173.3,170.1,82.1,67.1,66.8,60.1,57.3,56.0,55.0$, $45.9,43.9,42.5,42.3,41.8,41.7,40.7,40.2,38.3,34.9$, 29.7, 29.1, 28.1, 25.1, 21.9, 20.4, 19.1, 15.5, 14.4, 13.5; IR (KBr) $v$ : 2948, 2851, 1720, 1641, 1439, 1267, 1236, 1176, $1117 \mathrm{~cm}^{-1}$; HRMS calcd for $\mathrm{C}_{30} \mathrm{H}_{47} \mathrm{NO}_{6} 517.3403$, found 517.3414 .

3.2.8 16 $\beta$ - $O$-[4-氧亚基-4-(4-甲基-1-哌嗪基)-丁酰 基]-19-贝叶酸乙酯(IS-8b)的合成

按照合成 IS-2b 的方法由化合物 IS-7 (200.0 mg, $0.45 \mathrm{mmol}$ )、 $N$-甲基哌嗪 $(0.06 \mathrm{~mL}, 0.54 \mathrm{mmol}) 、$ DMAP 
(65.5 mg, $0.54 \mathrm{mmol}$ )和 EDCI (427.5 mg, $2.23 \mathrm{mmol}$ )反 应, 得 $175 \mathrm{mg}$ 白色固体产物 IS-8b, 产率 74\%. m.p. $88.2 \sim 88.9{ }^{\circ} \mathrm{C} ;[\alpha]_{\mathrm{D}}^{18}-44.3\left(c \quad 0.40, \mathrm{CH}_{2} \mathrm{Cl}_{2}\right) ;{ }^{1} \mathrm{H} \mathrm{NMR}$ $\left(\mathrm{CDCl}_{3}, 500 \mathrm{MHz}\right) \delta: 4.74 \sim 4.70(\mathrm{~m}, 1 \mathrm{H}, \mathrm{CHOCO})$, $4.12 \sim 4.01\left(\mathrm{~m}, 2 \mathrm{H}, \mathrm{OCH}_{2} \mathrm{CH}_{3}\right), 3.61(\mathrm{t}, J=6.1 \mathrm{~Hz}, 2 \mathrm{H}$, $\left.\mathrm{COCH}_{2}\right), 3.49\left(\mathrm{t}, J=6.3 \mathrm{~Hz}, 2 \mathrm{H}, \mathrm{CH}_{2} \mathrm{CO}\right), 2.69 \sim 2.34(\mathrm{~m}$, $8 \mathrm{H}$, piperazine $\mathrm{H}-2 \times 2, \mathrm{H}-3 \times 2, \mathrm{H}-5 \times 2, \mathrm{H}-6 \times 2), 2.30(\mathrm{~s}$, $\left.3 \mathrm{H}, \mathrm{NCH}_{3}\right), 2.14$ (d, $\left.J=15.8 \mathrm{~Hz}, \mathrm{H}_{\alpha}-15\right), 1.84 \sim 1.75(\mathrm{~m}$, $6 \mathrm{H}), 1.59 \sim 1.48(\mathrm{~m}, 4 \mathrm{H}), 1.40 \sim 1.33(\mathrm{~m}, 2 \mathrm{H}), 1.31(\mathrm{~d}, J=$ $3.3 \mathrm{~Hz}, 1 \mathrm{H}), 1.24(\mathrm{t}, J=8.9 \mathrm{~Hz}, 3 \mathrm{H}), 1.14(\mathrm{~s}, 3 \mathrm{H}), 1.05 \sim$ $0.99(\mathrm{~m}, 3 \mathrm{H}), 0.98 \sim 0.92(\mathrm{~m}, 1 \mathrm{H}), 0.84(\mathrm{~s}, 3 \mathrm{H}), 0.87 \sim$ $0.82(\mathrm{~m}, 1 \mathrm{H}), 0.71(\mathrm{~s}, 3 \mathrm{H}) ;{ }^{13} \mathrm{C} \mathrm{NMR}\left(\mathrm{CDCl}_{3}, 125 \mathrm{MHz}\right) \delta$ : $177.8,173.4,169.8,138.1,82.0,60.1,57.3,56.0,55.2$, $55.0,54.9,46.3,45.3,43.9,42.5,41.8,40.7,40.2,38.3$ (2C), 34.9, 29.8, 29.1, 28.3, 25.1, 21.9, 20.4, 19.1, 14.4, 13.5; IR (KBr) v: 2937, 2842, 1726, 1645, 1448, 1294, $1117 \mathrm{~cm}^{-1}$; HRMS calcd for $\mathrm{C}_{31} \mathrm{H}_{50} \mathrm{~N}_{2} \mathrm{O}_{5} 530.3721$, found 530.3731 .

$3.2 .916 \beta-O$ - $[4$ - 氧亚基-4-(4-叔丁氧羰基- 1 -哌嗪基 $)-$ 丁酰基]-19-贝叶酸乙酯(IS-9)的合成

按照合成 IS-3 的方法由化合物 IS-7 (360.3 mg, 0.80 mmol)、 $N$-Boc 哌嗪(179.4 mg, $0.96 \mathrm{mmol}) 、$ DMAP (117.7 $\mathrm{mg}, 0.96 \mathrm{mmol})$ 和 EDCI (769.2 mg, $4.01 \mathrm{mmol})$ 反应, 得 $350.1 \mathrm{mg}$ 无色液体产物 IS-9, 产率 71\%. $[\alpha]_{\mathrm{D}}^{20}-36.8$ (c $\left.0.42, \mathrm{CH}_{2} \mathrm{Cl}_{2}\right) ;{ }^{1} \mathrm{H} \mathrm{NMR}\left(\mathrm{CDCl}_{3}, 500 \mathrm{MHz}\right) \delta: 4.75 \sim 4.72$ (m, 1H, CHOCO), $4.14 \sim 4.01\left(\mathrm{~m}, 2 \mathrm{H}, \mathrm{CH}_{2} \mathrm{CH}_{3}\right), 3.58 \sim$ $3.40(\mathrm{~m}, 8 \mathrm{H}$, piperazine $\mathrm{H}-2 \times 2, \mathrm{H}-3 \times 2, \mathrm{H}-5 \times 2, \mathrm{H}-6 \times$ 2), $2.69 \sim 2.61\left(\mathrm{~m}, 4 \mathrm{H}, \mathrm{OCH}_{2} \mathrm{CH}_{2} \mathrm{O}\right), 2.15(\mathrm{~d}, J=13.2 \mathrm{~Hz}$, $\left.1 \mathrm{H}, \mathrm{H}_{\alpha}-15\right), 1.88 \sim 1.77(\mathrm{~m}, 5 \mathrm{H}), 1.69$ (t, $\left.J=13.1 \mathrm{~Hz}, 2 \mathrm{H}\right)$, $1.60 \sim 1.50(\mathrm{~m}, 4 \mathrm{H}), 1.47\left[\mathrm{~s}, 9 \mathrm{H}, \mathrm{C}\left(\mathrm{CH}_{3}\right)_{3}\right], 1.41 \sim 1.32(\mathrm{~m}$, $3 \mathrm{H}), 1.25(\mathrm{t}, J=7.1 \mathrm{~Hz}, 3 \mathrm{H}), 1.15(\mathrm{~s}, 3 \mathrm{H}), 1.06 \sim 0.94(\mathrm{~m}$, $4 \mathrm{H}), 0.90(\mathrm{~s}, 3 \mathrm{H}), 0.88 \sim 0.83(\mathrm{~m}, 1 \mathrm{H}), 0.72(\mathrm{~s}, 3 \mathrm{H}) ;{ }^{13} \mathrm{C}$ NMR $\left(\mathrm{CDCl}_{3}, 125 \mathrm{MHz}\right) \delta: 176.5,172.0,168.9,153.6$, $80.9,79.3,64.8,58.9,56.1,54.8,53.8,44.1,42.7,41.3$, 40.6 (2C), 39.5, 39.0, 37.1 (2C), 33.7, 28.5, 27.9, 27.4 (3C), 27.1, 23.9, 20.7, 19.2, 17.9 (2C), 14.3, 13.1, 12.3; IR (KBr) v: 2935, 2849, 1701, 1654, 1417, 1236, $1170 \mathrm{~cm}^{-1}$; HRMS calcd for $\mathrm{C}_{35} \mathrm{H}_{56} \mathrm{~N}_{2} \mathrm{O}_{7} 616.4088$, found 616.4079.

3.2.10 16 $\beta$ - $O$-[4-氧亚基-4-(1-哌嗪基)-丁酰基]-19贝叶酸乙酯(IS-10)的合成

按照合成 IS-4 的方法由化合物 IS-9 (100.0 mg, 0.16 $\mathrm{mmol})$ 和三氟乙酸 $(0.2 \mathrm{~mL}, 2.40 \mathrm{mmol})$ 反应, 得 $54.3 \mathrm{mg}$ 无色液体产物 IS-10, 产率 $65 \%$. $[\alpha]_{\mathrm{D}}^{20}-38.2$ (c 0.20 , $\left.\mathrm{CH}_{2} \mathrm{Cl}_{2}\right) ;{ }^{1} \mathrm{H} \mathrm{NMR}\left(\mathrm{CDCl}_{3}, 400 \mathrm{MHz}\right) \delta: 4.75 \sim 4.71(\mathrm{~m}$, $1 \mathrm{H}, \mathrm{CHOCO}), 4.13 \sim 4.02\left(\mathrm{~m}, 2 \mathrm{H}, \mathrm{CH}_{2} \mathrm{CH}_{3}\right), 3.72 \sim 2.93$ (m, $8 \mathrm{H}$, piperazine $\mathrm{H}-2 \times 2, \mathrm{H}-3 \times 2, \mathrm{H}-5 \times 2, \mathrm{H}-6 \times 2)$, $2.68 \sim 2.62\left(\mathrm{~m}, 4 \mathrm{H}, \mathrm{COCH}_{2} \mathrm{CH}_{2} \mathrm{CO}\right), 2.48(\mathrm{~d}, J=13.1 \mathrm{~Hz}$, $\left.\mathrm{H}_{\alpha^{-}}-15\right), 2.15\left(\mathrm{~d}, J=13.6 \mathrm{~Hz}, 1 \mathrm{H}, \mathrm{H}_{\beta^{-}}-15\right), 1.83 \sim 1.69(\mathrm{~m}$, $6 \mathrm{H}), 1.63 \sim 1.47(\mathrm{~m}, 5 \mathrm{H}), 1.41 \sim 1.32(\mathrm{~m}, 3 \mathrm{H}), 1.24(\mathrm{~s}$, $6 \mathrm{H}), 1.16(\mathrm{~s}, 3 \mathrm{H}), 1.06 \sim 0.97(\mathrm{~m}, 4 \mathrm{H}), 0.90(\mathrm{~s}, 3 \mathrm{H}), 0.87(\mathrm{t}$, $J=3.0 \mathrm{~Hz}, 1 \mathrm{H}), 0.72(\mathrm{~s}, 3 \mathrm{H}) ;{ }^{13} \mathrm{C} \mathrm{NMR}\left(\mathrm{CDCl}_{3}, 100 \mathrm{MHz}\right)$ $\delta: 189.2,177.5,173.0,169.7,81.8,81.7,59.8,57.0,55.6$, $54.7,45.0,44.7,43.6,42.2,41.4,40.9,40.4,39.8,38.0$, $34.5,29.6,29.4,28.8,27.8,24.8,21.6,20.1,18.8,14.0$, 13.1; IR (KBr) v: 2937, 2849, 1726, 1651, 1448, 1375, $1231,1175 \mathrm{~cm}^{-1}$; HRMS calcd for $\mathrm{C}_{30} \mathrm{H}_{48} \mathrm{~N}_{2} \mathrm{O}_{5} 516.3563$, found 516.3560 .

3.2.115-氧亚基-5-(16 $3-O-19$-氧亚基-19-乙氧基-贝 叶)戊酸(IS-11) 的合成

按照合成 IS-7 的方法由化合物 IS-6 (86.8 mg, 0.25 $\mathrm{mmol})$ 、戊二酸䣲 $(213.2 \mathrm{mg}, 1.75 \mathrm{mmol})$ 和 $\operatorname{DMAP}(36.6$ $\mathrm{mg}, 0.30 \mathrm{mmol}$ )反应, 得 $80.4 \mathrm{mg}$ 无色液体产物 IS-11, 产率 70\%。 $[\alpha]_{\mathrm{D}}^{21}-49.5\left(c \quad 0.38, \mathrm{CH}_{2} \mathrm{Cl}_{2}\right)$; ${ }^{1} \mathrm{H} \quad \mathrm{NMR}$ $\left(\mathrm{CDCl}_{3}, 400 \mathrm{MHz}\right) \delta: 4.75 \sim 4.71(\mathrm{~m}, 1 \mathrm{H}, \mathrm{CHOCO})$, $4.14 \sim 4.00\left(\mathrm{~m}, 2 \mathrm{H}, \mathrm{CH}_{2} \mathrm{CH}_{3}\right), 3.49 \sim 3.45(\mathrm{~m}, 2 \mathrm{H}$, $\mathrm{CH}_{2} \mathrm{CH}_{2} \mathrm{CH}_{2}$ ), 2.44 2.37 (m, 2H, $\mathrm{CH}_{2} \mathrm{CH}_{2} \mathrm{CH}_{2}$ ), 2.15 (d, $\left.J=13.1 \mathrm{~Hz}, \mathrm{H}_{\alpha}-15\right), 2.00 \sim 1.93(\mathrm{~m}, 2 \mathrm{H}), 1.85 \sim 1.69(\mathrm{~m}$, $6 \mathrm{H}), 1.60 \sim 1.50(\mathrm{~m}, 4 \mathrm{H}), 1.42 \sim 1.36(\mathrm{~m}, 2 \mathrm{H}), 1.32(\mathrm{~d}, J=$ $2.4 \mathrm{~Hz}, 1 \mathrm{H}), 1.23(\mathrm{t}, J=7.2 \mathrm{~Hz}, 3 \mathrm{H}), 1.16(\mathrm{~s}, 3 \mathrm{H}), 1.08 \sim$ $0.94(\mathrm{~m}, 4 \mathrm{H}), 0.90(\mathrm{~s}, 3 \mathrm{H}), 0.85$ (q, $J=9.7 \mathrm{~Hz}, 1 \mathrm{H}), 0.70$ $(\mathrm{s}, 3 \mathrm{H}) ;{ }^{13} \mathrm{C} \mathrm{NMR}\left(\mathrm{CDCl}_{3}, 100 \mathrm{MHz}\right) \delta: 177.5,176.6$, $172.1,80.8,64.8,59.0,56.1,54.7,53.8,42.7,41.4,40.5$, $39.7,39.0,37.1,33.6,32.5,32.0,27.9,23.9,20.7,19.2$, 19.0, 17.9, 14.2, 13.1, 12.2; IR (KBr) v: 3429, 2949, 2849, 1720, 1454, 1379, 1234, $1178 \mathrm{~cm}^{-1}$; HRMS calcd for $\mathrm{C}_{27} \mathrm{H}_{42} \mathrm{O}_{6} 462.2981$, found 462.3000 .

$3.2 .1216 \beta-O$-(5-氧亚基-5-吗啉基-丁酰基)-19-贝叶 酸乙酯(IS-12a) 的合成

按照合成 IS-2a 的方法由化合物 IS-11 (100.0 mg, $0.22 \mathrm{mmol}) 、$ 吗啉(0.02 mL, 0.26 mmol)、DMAP (31.6 mg, $0.26 \mathrm{mmol})$ 和 EDCI (207.0 mg, $1.08 \mathrm{mmol})$ 反应, 得 87.0 $\mathrm{mg}$ 无色液体产物 IS-12a, 产率 76\%. [ $\alpha]_{\mathrm{D}}^{20}-12.1(c$ $\left.0.24, \mathrm{CH}_{2} \mathrm{Cl}_{2}\right) ;{ }^{1} \mathrm{H} \mathrm{NMR}\left(\mathrm{CDCl}_{3}, 400 \mathrm{MHz}\right) \delta: 4.75 \sim 4.71$ (m, 1H, CHOCO), 4.12 4.00 (m, 2H, $\left.\mathrm{CH}_{2} \mathrm{CH}_{3}\right), 3.75 \sim$ $3.61(\mathrm{~m}, 8 \mathrm{H}$, morpholine $\mathrm{H}-2 \times 2, \mathrm{H}-3 \times 2, \mathrm{H}-5 \times 2, \mathrm{H}-6 \times$ 2), $3.49 \sim 3.45\left(\mathrm{~m}, 2 \mathrm{H}, \mathrm{CH}_{2} \mathrm{CH}_{2} \mathrm{CH}_{2}\right), 2.44 \sim 2.37(\mathrm{~m}, 4 \mathrm{H}$, $\left.\mathrm{CH}_{2} \mathrm{CH}_{2} \mathrm{CH}_{2}\right), 2.15$ (d, $\left.J=13.4 \mathrm{~Hz}, 1 \mathrm{H}, \mathrm{H}_{\alpha}-15\right), 1.98 \sim$ $1.93(\mathrm{~m}, 2 \mathrm{H}), 1.88 \sim 1.78(\mathrm{~m}, 4 \mathrm{H}), 1.60 \sim 1.56(\mathrm{~m}, 2 \mathrm{H})$, $1.54 \sim 1.48(\mathrm{~m}, 2 \mathrm{H}), 1.32(\mathrm{~d}, J=2.5 \mathrm{~Hz}, 1 \mathrm{H}), 1.27 \sim 1.22$ $(\mathrm{m}, 6 \mathrm{H}), 1.16(\mathrm{~s}, 3 \mathrm{H}), 1.08 \sim 1.01(\mathrm{~m}, 3 \mathrm{H}), 0.96(\mathrm{q}, J=9.4$ 
$\mathrm{Hz}, 1 \mathrm{H}), 0.90$ (s, 3H), 0.85 (q, $J=9.2 \mathrm{~Hz}, 1 \mathrm{H}), 0.70$ (s, $3 \mathrm{H}) ;{ }^{13} \mathrm{C} \mathrm{NMR}\left(\mathrm{CDCl}_{3}, 100 \mathrm{MHz}\right) \delta: 177.7,173.7,171.1$, 81.9, 67.1, 66.9, 60.1, 57.3, 55.9, 55.0, 46.1, 43.9, 42.6, $42.1,41.7,40.9,40.2,38.3,38.3,34.8,33.9,32.3,29.1$, 25.1, 21.9, 20.7, 20.4, 19.1, 18.6, 14.3, 13.5; IR (KBr) v: 2949, 2849, 1724, 1653, 1452, 1377, 1234, 1177, 1117 $\mathrm{cm}^{-1}$; HRMS calcd for $\mathrm{C}_{31} \mathrm{H}_{49} \mathrm{NO}_{6} 531.3560$, found 531.3566 .

3.2.13 16 3 - $O$ - $[5$ - 氧亚基-5-(4-甲基-1-哌嗪基)-戊酰 基]-19-贝叶酸乙酯(IS-12b)的合成

按照合成 IS-2b 的方法由化合物 IS-11 (200.0 mg, $0.43 \mathrm{mmol}$ )、 $N$-甲基哌嗪 $(0.06 \mathrm{~mL}, 0.52 \mathrm{mmol}$ )、DMAP (63.3 mg, $0.52 \mathrm{mmol}$ )和 EDCI (414.1 mg, $2.16 \mathrm{mmol})$ 反 应, 得 $169.2 \mathrm{mg}$ 无色液体产物 IS-12b, 产率 72\%. [ $\alpha]_{\mathrm{D}}^{20}$ -34.2 (c 0.44, $\left.\mathrm{CH}_{2} \mathrm{Cl}_{2}\right)$; ${ }^{1} \mathrm{H}$ NMR $\left(\mathrm{CDCl}_{3}, 500 \mathrm{MHz}\right) \delta$ : $4.73(\mathrm{~d}, J=6.2 \mathrm{~Hz}, 1 \mathrm{H}, \mathrm{CHOCO}), 4.12 \sim 4.02(\mathrm{~m}, 2 \mathrm{H}$, $\left.\mathrm{CH}_{2} \mathrm{CH}_{3}\right), 3.75 \sim 3.48\left(\mathrm{~m}, 6 \mathrm{H}, \mathrm{CH}_{2} \mathrm{CH}_{2} \mathrm{CH}_{2}\right), 2.44 \sim 2.37$ (m, $8 \mathrm{H}$, piperazine $\mathrm{H}-2 \times 2, \mathrm{H}-3 \times 2, \mathrm{H}-5 \times 2, \mathrm{H}-6 \times 2$ ), 2.31 (s, $\left.3 \mathrm{H}, \mathrm{NCH}_{3}\right), 2.15$ (d, $\left.J=13.4 \mathrm{~Hz}, \mathrm{H}_{\alpha}-15\right), 2.00 \sim$ $1.92(\mathrm{~m}, 2 \mathrm{H}), 1.84 \sim 1.69(\mathrm{~m}, 6 \mathrm{H}), 1.55(\mathrm{q}, J=15.0 \mathrm{~Hz}$, $4 \mathrm{H}), 1.34(\mathrm{~d}, J=14.3 \mathrm{~Hz}, 2 \mathrm{H}), 1.27 \sim 1.23(\mathrm{~m}, 3 \mathrm{H}), 1.16$ (s, 3H), $1.07 \sim 0.97$ (m, 4H), 0.90 (s, 3H), 0.84 (t, $J=16.6$ $\mathrm{Hz}, 1 \mathrm{H}), 0.70(\mathrm{~s}, 3 \mathrm{H}) ;{ }^{13} \mathrm{C} \mathrm{NMR}\left(\mathrm{CDCl}_{3}, 125 \mathrm{MHz}\right) \delta$ : 177.7, 173.7, 170.8, 81.8, 60.1, 57.3, 55.9, 55.4, 55.0, 54.9, $46.2,45.6,43.9,42.6,41.8,41.7,40.9,40.2,38.3,38.2$, $34.8,34.0,33.3,32.5,29.1,25.1,21.9,20.8,20.4,19.1$, 14.3, 13.5; IR (KBr) v: 2941, 2845, 2793, 1726, 1651, 1454, 1375, 1292, 1229, $1150 \mathrm{~cm}^{-1}$; HRMS calcd for $\mathrm{C}_{32} \mathrm{H}_{52} \mathrm{~N}_{2} \mathrm{O}_{5} 544.3876$, found 544.3879.

3.2.14 16 3 - $O$ - $[5$ - 氧亚基-5-(4-叔丁氧羰基-1-哌嗪 基)-戊酰基]-19-贝叶酸乙酯(IS-13)的合成

按照合成 IS-3 的方法由化合物 IS-11 (100.0 mg, $0.22 \mathrm{mmol}) 、 N$-Boc 哌嗪(48.3 mg, $0.26 \mathrm{mmol}) 、$ DMAP (31.7 mg, $0.26 \mathrm{mmol}$ )和 EDCI $(207.2 \mathrm{mg}, 1.08 \mathrm{mmol}) 反$ 应, 得 $98.0 \mathrm{mg}$ 无色液体产物 IS-13, 产率 $72 \%$. [ $\alpha]_{\mathrm{D}}^{22}$ -31.9 (c 0.48, $\left.\mathrm{CH}_{2} \mathrm{Cl}_{2}\right)$; ${ }^{1} \mathrm{H}$ NMR $\left(\mathrm{CDCl}_{3}, 400 \mathrm{MHz}\right) \delta$ : $4.75 \sim 4.72(\mathrm{~m}, 1 \mathrm{H}, \mathrm{CHOCO}), 4.14 \sim 4.00(\mathrm{~m}, 2 \mathrm{H}$, $\mathrm{CH}_{2} \mathrm{CH}_{3}$ ), $3.75 \sim 3.68\left(\mathrm{~m}, 2 \mathrm{H} \mathrm{CH} \mathrm{CH}_{2} \mathrm{CH}_{2}\right), 3.58 \sim 3.40$ (m, $8 \mathrm{H}$, piperazine $\mathrm{H}-2 \times 2, \mathrm{H}-3 \times 2, \mathrm{H}-5 \times 2, \mathrm{H}-6 \times 2$ ), $2.44 \sim 2.37\left(\mathrm{~m}, 4 \mathrm{H}, \mathrm{CH}_{2} \mathrm{CH}_{2} \mathrm{CH}_{2}\right), 2.15(\mathrm{~d}, J=13.5 \mathrm{~Hz}$, $\left.\mathrm{H}_{\alpha}-15\right), 2.00 \sim 1.93(\mathrm{~m}, 2 \mathrm{H}), 1.84 \sim 1.75(\mathrm{~m}, 4 \mathrm{H}), 1.59$ (q, $J=13.0 \mathrm{~Hz}, 3 \mathrm{H}), 1.47$ (s, 9H), 1.34 (q, $J=2.8 \mathrm{~Hz}, 2 \mathrm{H}$ ), $1.26 \sim 1.23(\mathrm{~m}, 6 \mathrm{H}), 1.16(\mathrm{~s}, 3 \mathrm{H}), 1.08 \sim 1.01(\mathrm{~m}, 3 \mathrm{H})$, 0.96 (q, $J=9.4 \mathrm{~Hz}, 1 \mathrm{H}), 0.90(\mathrm{~s}, 3 \mathrm{H}), 0.87 \sim 0.83(\mathrm{~m}, 1 \mathrm{H})$, $0.70(\mathrm{~s}, 3 \mathrm{H}) ;{ }^{13} \mathrm{C} \mathrm{NMR}\left(\mathrm{CDCl}_{3}, 100 \mathrm{MHz}\right) \delta: 177.7,173.7$,
171.1, 154.8, 81.9, 80.5, 60.1, 58.7, 57.3, 55.9, 55.0, 53.6, $45.5,43.9,42.6,41.7,41.6,40.9,40.2,38.3,38.3,34.8$, 33.9, 32.6, 29.1, 28.6 (3C), 25.2, 21.9, 20.7, 20.4, 19.1, $18.7,14.3,13.5$; IR (KBr) v: 2939, 2851, 1726, 1653, $1458,1420,1238,1173 \mathrm{~cm}^{-1}$; HRMS calcd for $\mathrm{C}_{36} \mathrm{H}_{58} \mathrm{~N}_{2-}$ $\mathrm{O}_{7} 630.4244$, found 630.4243 .

3.2.15 16 $\beta$ - $O$-[5-氧亚基-5-(1-哌嗪基)-戊酰基]-19贝叶酸乙酯(IS-14)的合成

按照合成 IS-4 的方法由化合物 IS-13 (50.0 mg, 0.07 $\mathrm{mmol})$ 和三氟乙酸 $(0.2 \mathrm{~mL}, 2.10 \mathrm{mmol})$ 反应得 $69.0 \mathrm{mg}$ 淡 黄色液体 IS-14, 产率 $82 \%$. $[\alpha]_{\mathrm{D}}^{20}-30.0$ (c 0.04 , $\left.\mathrm{CH}_{2} \mathrm{Cl}_{2}\right) ;{ }^{1} \mathrm{H}$ NMR $\left(\mathrm{CDCl}_{3}, 400 \mathrm{MHz}\right) \delta: 4.75 \sim 4.71(\mathrm{~m}$, $1 \mathrm{H}, \mathrm{CHOCO}), 4.14 \sim 4.00\left(\mathrm{~m}, 2 \mathrm{H}, \mathrm{CH}_{2} \mathrm{CH}_{3}\right), 3.69 \sim 2.92$ (m, $8 \mathrm{H}$, piperazine $\mathrm{H}-2 \times 2, \mathrm{H}-3 \times 2, \mathrm{H}-5 \times 2, \mathrm{H}-6 \times 2$ ), $2.47 \sim 2.38\left(\mathrm{~m}, 6 \mathrm{H}, \mathrm{CH}_{2} \mathrm{CH}_{2} \mathrm{CH}_{2} \mathrm{CO}\right), 2.15(\mathrm{~d}, J=13.6 \mathrm{~Hz}$, $\left.\mathrm{H}_{\alpha}-15\right), 1.85 \sim 1.72(\mathrm{~m}, 2 \mathrm{H}), 1.64 \sim 1.50(\mathrm{~m}, 4 \mathrm{H}), 1.42 \sim$ $1.32(\mathrm{~m}, 3 \mathrm{H}), 1.27 \sim 1.23(\mathrm{~m}, 3 \mathrm{H}), 1.16(\mathrm{~s}, 3 \mathrm{H}), 1.08 \sim$ 0.99 (m, 3H), 0.96 (q, $J=9.3 \mathrm{~Hz}, 1 \mathrm{H}), 0.90$ (s, 3H), 0.85 (q, $J=9.5 \mathrm{~Hz}, 1 \mathrm{H}), 0.70(\mathrm{~s}, 3 \mathrm{H}) ;{ }^{13} \mathrm{C}$ NMR $\left(\mathrm{CDCl}_{3}, 100\right.$ MHz) $\delta$ : 177.1, 173.1, 170.3, 81.2, 59.5, 56.6, 55.2, 54.3, $45.3,44.9,43.3,41.9,41.2,41.1$ (2C), 40.3, 39.5, 37.6, $37.6,34.2,33.3,31.8,29.3,28.5,24.5,21.3,20.1,19.8$, $18.5,13.7,12.9$; IR (KBr) v: 2947, 2849, 1724, 1651, 1452, 1377, $1178 \mathrm{~cm}^{-1}$; HRMS calcd for $\mathrm{C}_{31} \mathrm{H}_{50} \mathrm{~N}_{2} \mathrm{O}_{5}$ 530.3720 , found 530.3713 .

3.2.16 贝叶-15-烯-19-酸乙酯(IS-16)的合成

参考文献[19], 在圆底烧瓶中加入 2,4,6-三甲基吡 啶 (5 mL), 将 IS-15 (690.0mg, $1.37 \mathrm{~mol}$ ) 溶于其中, 回流 反应 $4 \mathrm{~h}$, 经薄层层析检测反应结束后, 减压蒸馏除去 溶剂, 用乙酸乙酯和水萃取, 并用 $1 \mathrm{~mol} / \mathrm{L} \mathrm{HCl}$ 洗涤至微 酸性, 用饱和氯化钠洗涤, 过滤, 柱层析得到无色液体 IS-16, 产率 95\%. $[\alpha]_{\mathrm{D}}^{22}-5.0\left(c 0.04, \mathrm{CH}_{2} \mathrm{Cl}_{2}\right) ;{ }^{1} \mathrm{H} \mathrm{NMR}$ $\left(\mathrm{CDCl}_{3}, 400 \mathrm{MHz}\right) \delta: 5.71,5.45(\mathrm{~d}, J=5.5 \mathrm{~Hz}$, each $1 \mathrm{H}$, $\mathrm{H}-15, \mathrm{H}-16), 4.10$ (q, $\left.J=14.12 \mathrm{~Hz}, \mathrm{OCH}_{2} \mathrm{CH}_{3}\right), 2.16$ (d, $J=13.1 \mathrm{~Hz}, 1 \mathrm{H}), 1.79(\mathrm{t}, J=14.4 \mathrm{~Hz}, 3 \mathrm{H}), 1.69 \sim 1.64(\mathrm{~m}$, $3 \mathrm{H}), 1.57 \sim 1.51(\mathrm{~m}, 1 \mathrm{H}), 1.46 \sim 1.37(\mathrm{~m}, 3 \mathrm{H}), 1.29 \sim 1.25$ (m, 6H), $1.21(\mathrm{~d}, J=1.6 \mathrm{~Hz}, 1 \mathrm{H}), 1.18(\mathrm{~s}, 3 \mathrm{H}), 1.08(\mathrm{dd}$, $J=2.2,11.4 \mathrm{~Hz}, 1 \mathrm{H}), 1.02$ (s, 1H), 1.00 (s, 3H), 0.88 (q, $1 \mathrm{H}), 0.59(\mathrm{~s}, 3 \mathrm{H}) ;{ }^{13} \mathrm{C} \mathrm{NMR}\left(\mathrm{CDCl}_{3}, 100 \mathrm{MHz}\right) \delta: 177.7$, 136.7, 135.0, 61.2, 60.1, 57.3, 52.4, 49.3, 44.0, 43.8, 39.8, $38.4,38.0,37.9,33.3,29.3,25.1,21.8,20.6,19.5,14.3$, 14.1; IR (KBr) v: 3035, 2945, 2847, 1724, 1452, 1232 $\mathrm{cm}^{-1}$; HRMS calcd for $\mathrm{C}_{22} \mathrm{H}_{34} \mathrm{O}_{2} 330.2559$, found 330.2551 . 


\subsubsection{7 贝叶-15-烯-19-酸(IS-17)的合成}

参考文献[19], 在圆底烧瓶中加入 2,4,6-三甲基吡 啶 $(7 \mathrm{~mL})$, 将 IS-16 (375.1 mg, $1.13 \mathrm{~mol})$ 和碘化锂(455.7 $\mathrm{mg}, 3.40 \mathrm{mmol}$ ) 溶于其中, 回流反应 $12 \mathrm{~h}$, 经薄层层析 检测反应结束后, 用乙酸乙酯和水萃取, 并用 $1 \mathrm{~mol} / \mathrm{L}$ $\mathrm{HCl}$ 洗涤至微酸性, 用饱和氯化钠洗涤, 过滤, 柱层析 得到白色固体产物, 产率 $90 \%$. m.p. 177.4 $177.9{ }^{\circ} \mathrm{C}$. $[\alpha]_{\mathrm{D}}^{20}-6.4\left(c 0.39, \mathrm{CH}_{2} \mathrm{Cl}_{2}\right) ;{ }^{1} \mathrm{H} \mathrm{NMR}\left(\mathrm{CDCl}_{3}, 400 \mathrm{MHz}\right)$ $\delta: 5.74,5.46$ (d, $J=5.6 \mathrm{~Hz}$, each $1 \mathrm{H}, \mathrm{H}-15, \mathrm{H}-16), 2.15$ (d, $J=13.2 \mathrm{~Hz}, 1 \mathrm{H}), 1.82 \sim 1.74(\mathrm{~m}, 4 \mathrm{H}), 1.70 \sim 1.58(\mathrm{~m}, 3 \mathrm{H})$, $1.55 \sim 1.33(\mathrm{~m}, 5 \mathrm{H}), 1.29(\mathrm{~d}, J=4.5 \mathrm{~Hz}, 2 \mathrm{H}), 1.25(\mathrm{~s}, 3 \mathrm{H})$, $1.13 \sim 1.02(\mathrm{~m}, 3 \mathrm{H}), 1.00(\mathrm{~s}, 3 \mathrm{H}), 0.89(\mathrm{~d}, J=9.3 \mathrm{~Hz}, 1 \mathrm{H})$, $0.67(\mathrm{~s}, 3 \mathrm{H}) ;{ }^{13} \mathrm{C} \mathrm{NMR}\left(\mathrm{CDCl}_{3}, 100 \mathrm{MHz}\right) \delta: 184.8,136.7$, 135.0, 61.2, 57.3, 52.5, 49.3, 44.0, 43.8, 39.7, 38.2, 38.1, 37.8, 33.3, 29.3, 25.1, 21.8, 20.7, 19.4, 14.0; IR (KBr) v: 3030, 2999, 2941, 2850, 1690, 1448, $1256 \mathrm{~cm}^{-1}$; HRMS calcd for $\mathrm{C}_{20} \mathrm{H}_{30} \mathrm{O}_{2}$ 302.2246, found 302.2248.

\section{3 体外抗肿瘤细胞增殖实验}

\subsection{1 实验材料}

细胞系: 人皮肤鳞癌细胞(Colo-16)和人肺腺癌细胞 (A549).

材料: 胎牛血清(Hyclone)、RPMI1640 培养基 (Gibco)、青霉素、链霉素双抗、胰酶-EDTA 溶液、磷酸 缓冲液(PBS)、噻唑蓝(MTT)、二甲基亚砜(DMSO)、细 胞培养瓶及 96 孔细胞培养板、倒置显微镜(CKX41, Olympus)、二氧化碳细胞培养箱(Thermo)、超净工作台、 离心机(Centrifuge 5804 R, eppendorf)、酶标仪(xMark, BIO-RAD)及待测的药品.

\subsection{2 实验方法}

配置待测的药品: 将本实验待测样品和阳性对照药 品阿霉素、齐墩果酸和氟尿嘧啶溶解于安全剂量的 DMSO $50 \mu \mathrm{L}$ 中, 后用质量分数为 $10 \%$ RPMI1640 完全 培养液将待测药品分别配成 $320,160,80,40$ 和 20 $\mu \mathrm{mol} \cdot \mathrm{L}^{-1} 5$ 个剂量, 相应的终浓度分别 $160,80,40,20$ 和 $10 \mu \mathrm{mol} \cdot \mathrm{L}^{-1}$.

抗肿瘤细胞增殖实验: 将 Colo-16 和 A549细胞分别 在含质量分数为 $10 \%$ 胎牛血清的 RPMI1640 培养液中扩 增细胞, 待细胞铺满瓶底约 $80 \%$ 时更换新鲜培养液, 于 第二天用质量分数为 $0.25 \%$ 肤酶-EDTA 溶液消化贴壁细 胞并收集、洗涤细胞, 将 Colo-16 和 A549 细胞的细胞浓 度均调整为 $2 \times 10^{4}$ 个 $/ \mathrm{mL}$. 将二种细胞各自分别接种 于 96 孔细胞培养板中, $100 \mu \mathrm{L} /$ 孔, 置细胞板于体积分数 为 $5 \% \mathrm{CO}_{2}$ 细胞培养箱中孵育 $24 \mathrm{~h}$, 之后分别取 5 个剂 量的待测药品 $100 \mu \mathrm{L} /$ 孔加到细胞板中, 每个浓度设三 个复孔. 振荡器振荡细胞板 $30 \mathrm{~s}$, 随后置细胞培养箱中
孵育 $48 \mathrm{~h}$. 在终止实验前 $4 \mathrm{~h}$, 加 MTT $20 \mu \mathrm{L}$ / 孔, 继续孵 育, 实验终止时, 将细胞板在 $4{ }^{\circ} \mathrm{C}, 2000 \mathrm{r} / \mathrm{min}$ 离心 10 $\min$, 甩板后加入 DMSO $150 \mu \mathrm{L} /$ 孔, 测定 $570 \mathrm{~nm}$ 下的 $\mathrm{OD}$ 值. 获得数据后, 采用 SPSS17.0 软件计算 $\mathrm{IC}_{50}$.

\section{4 抑菌实验}

参考文献[20], 将 E. coli(大肠杆菌)1924、S. aureus (金黄色葡萄球菌)4220、S. mutans(变形链球菌)3289、 $\operatorname{MRSA}$ (耐甲氧西林金黄色葡萄球菌)3167 和 QRSA(耐喹 诺酮金黄色葡萄球菌)3505 五种菌种稀释 1000 倍后, 各 自在十个 96 孔微量滴定板上接种, 保持恒温 $37{ }^{\circ} \mathrm{C}$, 培 养 $24 \mathrm{~h}$, 加入质量分数为 $0.05 \%$ 的所测样品溶液. 继续 培养 $24 \mathrm{~h}$ 后，使用酶联免疫吸收测定仪对菌种的生长情 况进行检测，以对其最小抑菌浓度(MIC)进行测试. 采 用相同的方法用氯霉素(Chloramphenicol)做对照试验.

辅助材料(Supporting Information) 目标化合物 ${ }^{1} \mathrm{H}$ $\mathrm{NMR},{ }^{13} \mathrm{C} \mathrm{NMR}$ 和 HRMS 图谱. 这些材料可以免费从本 刊网站(http://sioc-journal.cn/)上下载.

\section{References}

[1] Lin, J.; Guo, Y.; Gao, Y.; Wang, S.; Wang, X.; Xie, Z.; Niu, H.; Chang, W.; Liu, L.; Yuan, H.; Lou, H. J. Med. Chem. 2015, 58, 3944.

[2] Liu, C. J.; Yu, S. L.; Liu, Y. P.; Dai, X. J.; Wu, Y.; Li, R. J.; Tao, J. C. Eur. J. Med. Chem. 2016, 115, 26.

[3] Wang, M.; Li, H.; Xu, F.; Gao, X.; Li, J.; Xu, S.; Zhang, D.; Wu, X.; Xu, J.; Hua, H.; Li, D. Eur. J. Med. Chem. 2018, 156, 885.

[4] Luan, T.; Cao, L. H.; Deng, H.; Shen, Q. K.; Tian, Y. S.; Quan, Z. S. Molecules 2019, 24, 121.

[5] Wu, Y.; Liu, C. J.; Liu, X.; Dai, G. F.; Du, J. Y.; Tao, J. C. Helv Chim. Acta 2010, 93, 2052.

[6] Hsieh, M. H.; Chan, P.; Sue, Y. M.; Liu, J. C.; Liang, T. H.; Huang, T. Y.; Tomlinson, B.; Chow, M.; Kao, P. F.; Chen, Y. J. Clin Ther. 2003, 25, 2797.

[7] Ma, J.; Ma, Z.; Wang, J.; Milne, R. W.; Xu, D.; Davey, A. K.; Evans, A. M. Diabetes, Obes. Metab. 2007, 9, 597.

[8] Samadder A.; Das, J.; Das, S.; Khuda, A. R. Environ. Toxicol. Pharmacol. 2012, 34, 743.

[9] Jin H.; Gerber J. P.; Wang J.; Ji, M.; Davey, A. K. J. Pharm. Biomed. Anal. 2008, $48,986$.

[10] Wong, K. L.; Lin, J. W.; Liu, J. C.; Yang, H. Y.; Kao, P. F.; Chen, C. H.; Loh, S. H.; Chiu, W. T.; Cheng, T. H.; Lin, J. G.; Hong, H. J. Pharmacology 2006, 76, 163.

[11] Hutt, O. E.; Doan, T. L.; Georg, G. I. Org. Lett. 2013, 15, 1602.

[12] Zhu, S. L.; Wu, Y.; Liu, C. J.; Wei, C. Y.; Tao, J. C.; Liu, H. M. Eur. J. Med. Chem. 2013, 65, 70.

[13] Tian, T.; Liu, X.; Lee, E. S.; Sun, J.; Feng, Z.; Zhao, L.; Zhao, C. Arch. Pharmacal Res. 2017, 40, 458.

[14] Chen, Y. H.; Hou, X. Y.; Zhi, D. F.; Li, C.; Tian, T.; Sun, J. Y.; Zhao, L. X.; Zhao, C. H. Chin. J. Org. Chem. 2016, 36, 795 (in Chinese).

(陈艳华, 侯熙彦, 支德福, 李常, 田甜, 孙竞阳, 赵龙铉, 赵春 晖, 有机化学, 2016, 36, 795.)

[15] Liu, X. Y.; Gao, X. H. Q.; Jin, X. J.; Zhao, C. H.; Feng, Z. H.; Sui, Y.; Zhao, L. X.; Yan, X. Chin. J. Org. Chem. 2018, 38, 3227 (in Chinese).

(刘新宇, 高雪琴, 金学军, 赵春晖, 冯中华, 隋悦, 赵龙铉, 阎 欣, 有机化学, 2018, 38, 3227.) 
[16] Lohoelter, C.; Weckbecker, M.; Waldvogel, S. R. Eur. J. Org. Chem. 2013, 2013, 5539.

[17] Zhang, T.; Lu, L. H.; Liu, H.; Wang, J. W.; Wang, R. X.; Zhang, Y. X.; Tao, J. C. Bioorg. Med. Chem. Lett. 2012, 22, 5827.

[18] Liu, C. J.; Liu, Y. P.; Yu, S. L.; Dai, X. J.; Zhang, T.; Tao, J. C. Bioorg. Med. Chem. Lett. 2016, 26, 5455.

[19] Murillo, J. A.; Gil, J. F.; Upegui, Y. A.; Restrepo, A. M.; Robledo,
S. M.; Quiñones, W.; Echeverri, F.; Martin, A. S.; Olivo, H. F.; Escobar, G. Bioorg. Med. Chem. 2019, 27, 153.

[20] Xing, X. L.; Liu, T. T.; Song, M. X.; Li, Y. R.; Zhang, Y.; Li, Y.; Li, H. S.; Zheng, C. J.; Piao, H. R. Chin. J. Med. 2013, 23, 1 (in Chinese).

(邢肖兰, 刘婷婷, 宋明霞, 李亚茹, 张雨, 李燕, 李花淑, 郑昌 吉，朴虎日，中国药物化学杂志, 2013, 23, 1.) 